\title{
Skycorr: A general tool for spectroscopic sky subtraction
}

\author{
S. Noll ${ }^{1}$, W. Kausch ${ }^{1,2}$, S. Kimeswenger ${ }^{3,1}$, M. Barden ${ }^{4}$, A. M. Jones ${ }^{1}$, A. Modigliani ${ }^{5}$, C. Szyszka ${ }^{1}$, and J. Taylor ${ }^{5}$ \\ ${ }^{1}$ Institute for Astro- and Particle Physics, University of Innsbruck, Technikerstr. 25/8, 6020 Innsbruck, Austria \\ e-mail: stefan.noll@uibk.ac.at \\ 2 Department of Astrophysics, University of Vienna, Türkenschanzstrasse 17, 1180 Vienna, Austria \\ 3 Instituto de Astronomía, Universidad Católica del Norte, Avenida Angamos 0610, Antofagasta, Chile \\ ${ }^{4}$ International Graduate School of Science and Engineering, Technische Universität München, Boltzmannstr. 17, \\ 85748 Garching bei München, Germany \\ 5 European Southern Observatory, Karl-Schwarzschild-Str. 2, 85748 Garching bei München, Germany
}

Received 30 March 2014 / Accepted 9 May 2014

\begin{abstract}
Context. Airglow emission lines, which dominate the optical-to-near-infrared sky radiation, show strong, line-dependent variability on time scales from minutes to decades. Therefore, the subtraction of the sky background in the affected wavelength regime becomes a problem if plain-sky spectra have to be taken at a different time from the astronomical data.

Aims. A solution of this problem is the physically motivated scaling of the airglow lines in the plain-sky data to fit the sky lines in the object spectrum. We have developed a corresponding instrument-independent approach based on one-dimensional spectra.

Methods. Our code skycorr separates sky lines and sky/object continuum by an iterative approach involving a line finder and airglow line data. The sky lines, which mainly belong to $\mathrm{OH}$ and $\mathrm{O}_{2}$ bands, are grouped according to their expected variability. The line groups in the sky data are then scaled to fit the sky in the science data. Required pixel-specific weights for overlapping groups are taken from a comprehensive airglow model. Deviations in the wavelength calibration are corrected for by fitting Chebyshev polynomials and rebinning via asymmetric damped sinc kernels. The scaled sky lines and the sky continuum are subtracted separately.

Results. ESO-VLT X-shooter data covering $2.5 \mathrm{~h}$ with a good time resolution were selected to illustrate the performance. Data taken six nights and about one year before were also used as reference sky data. The variation of the sky-subtraction quality as a function of time difference between the object and sky data depends on changes in the airglow intensity, atmospheric transparency, and instrument calibration. Except for short time intervals of a few minutes, the sky line residuals were between 2.1 and 5.5 times weaker than for sky subtraction without fitting. Additional tests showed that skycorr performs consistently better than the method of Davies (2007, MNRAS, 375, 1099) developed for ESO-VLT SINFONI data.
\end{abstract}

Key words. atmospheric effects - radiation mechanisms: non-thermal - instrumentation: spectrographs - methods: data analysis methods: numerical - techniques: spectroscopic

\section{Introduction}

For spectroscopic instrument set-ups and observing programmes that do not provide plain-sky spectra simultaneously with the desired object spectra (two-dimensional (2D) spectra where the object only covers a small fraction in spatial direction would be ideal), it is necessary to use sky spectra taken at a different time from the science spectra for the sky correction. This especially affects fiber spectrographs, which include instruments using an integral-field unit (IFU). The strong variability of airglow emission on various time scales from minutes to decades (see e.g. Khomich et al. 2008; Patat 2008; Noll et al. 2012) can cause unavoidable sky-correction residua. Significant changes in the airglow emission can also be expected for angular distances in the order of a few degrees or even less depending on the characteristics of possible wave patterns, such as those caused by internal gravity waves (see e.g. Taylor et al. 1997). Moreover, different telescope positions and ambient conditions may cause instrument flexures, which result in wavelength shifts of the sky spectrum with respect to the science spectrum. Hence, the reference sky spectrum has to be adapted in both the flux and the wavelength regime to allow a reasonable sky-background correction.

An illustration of this problem and a solution for the Very Large Telescope (VLT) near-infrared (near-IR) integral-field spectrograph SINFONI (Eisenhauer et al. 2003) of the European Southern Observatory (ESO) has been described by Davies (2007). His airglow correction method is based on using arbitrary sky spectra for the sky subtraction in science data by scaling physically related line groups of the sky spectrum to the corresponding groups in the science spectrum. Subsequently, the newly created scaled spectrum is used for the sky correction. Specifically, Davies' method groups emission lines from vibrational-rotational transitions resulting from non-thermal excitation processes of the $\mathrm{OH}$ molecule (Meinel 1950; Bates \& Nicolet 1950; Rousselot et al. 2000; Khomich et al. 2008) and defines wavelength regions where these groups dominate the airglow emission. These wavelength ranges are scaled in the sky spectrum to match the corresponding flux in the object spectrum. This approach only works for the line component of the sky emission. Since object and sky continua cannot satisfyingly be separated, the sky continuum cannot be adapted and has to be subtracted before the scaling procedure for the line emission can start. Davies' code considers a greybody for the thermal continuum, which is fitted to the data. Other continuum sources that dominate the emission at wavelengths shorter than the $K$ band (e.g. airglow (pseudo-)continua, scattered moonlight, zodiacal light, and instrument-related continua; see Noll et al. 2012) are not taken into account. In the $J$ and $H$ bands covered by the SINFONI data, these contributions are distinctly fainter than the 
Sky spectrum

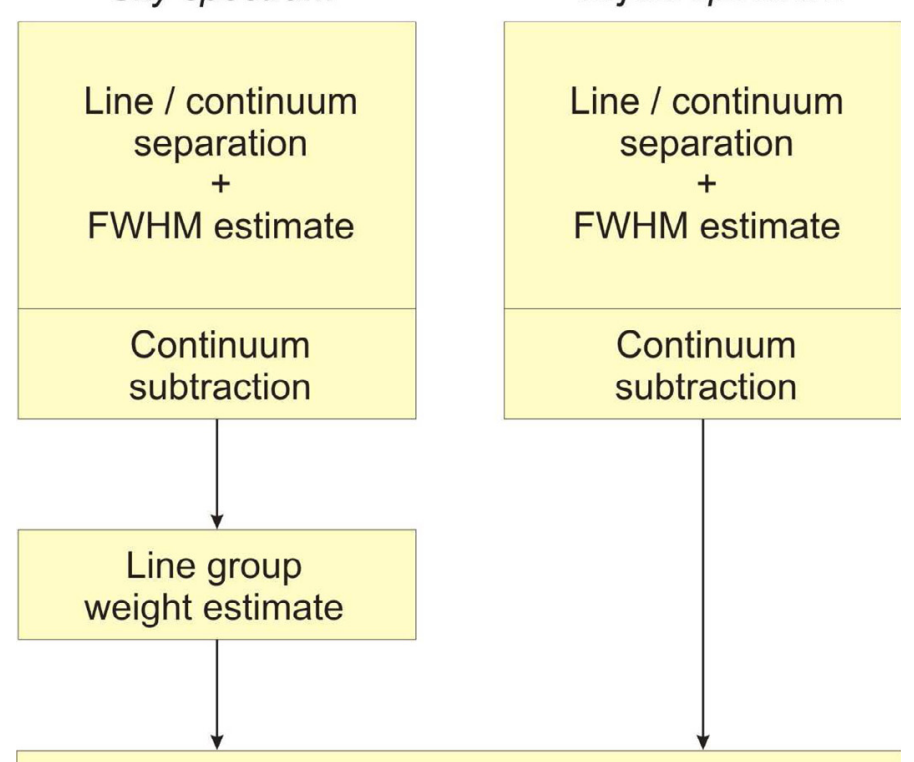

Fitting of $\lambda$ and line flux (mpfit)

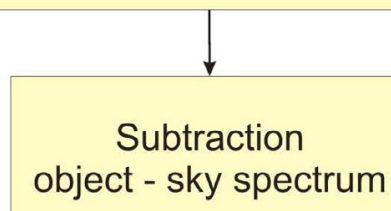

Fig. 1. Overview of the skycorr workflow.

airglow lines. Since Davies' method is restricted to $\mathrm{OH}$ airglow lines beyond $1 \mu \mathrm{m}^{1}$, it can only be applied to near-IR wavelength regions where other line emission is negligible. Davies' method has successfully been implemented for the ESO SINFONI datareduction pipeline (Modigliani et al. 2007).

In this paper, we present the instrument-independent code skycorr ${ }^{2}$ for the sky correction based on one-dimensional (1D) spectra. It was inspired by Davies' pioneering method. In comparison, skycorr allows a more accurate subtraction of airglow lines because of its more detailed airglow model (see Noll et al. 2012 for the optical range), the pixel-specific scaling of the lines, and an advanced approach for separating lines and continuum. Moreover, the code can also be applied to optical wavelengths. Section 2 describes the algorithm implemented in skycorr. A test data set for illustrating the performance is presented in Sect. 3. The test results are discussed in Sect. 4. This also involves a comparison with Davies' method. Finally, Sect. 5 provides a summary and contains our conclusions.

\section{Method and use}

In the following, we describe the skycorr sky-correction procedure in detail. The algorithm is sketched in Fig. 1. Its main

\footnotetext{
1 Version 2.0 of Davies' code also allows simple scaling of the strong $\mathrm{O}_{2}$ band at $1.27 \mu \mathrm{m}$. This feature is not described in Davies (2007).

2 The code can be downloaded from web pages located at ESO (http://www.eso.org/pipelines/skytools/) as well as from the University of Innsbruck (http://www.uibk.ac.at/eso/ software/).
}

purpose is the removal of sky emission lines in a science spectrum by means of a scaled reference sky spectrum. Any continuum component in the input science and reference sky spectra has to be removed in advance. Hence, as a first step, pixels belonging to lines and continuum have to be identified, separated, and masked accordingly. The line identification is discussed in Sect. 2.1. This procedure also derives the typical line full width at half maximum (FWHM, Sect. 2.2). The next step is a fit of the continuum pixels alone, which is subtracted from the input spectrum to obtain a continuum-free spectrum (Sect. 2.3). In the third step, a weight mask for the different airglow line groups in the reference sky spectrum is calculated, which incorporates an extended version of the sky-radiance model described in Noll et al. (2012) (Sect. 2.4). It is an essential input for scaling the reference sky line spectrum to fit the science line spectrum in the subsequent step (Sect. 2.5). The fitting procedure also allows an optimisation of the wavelength grids (Sect. 2.6). The final step of the sky-correction procedure is the sky subtraction itself. This operation is just the subtraction of the best-fit sky line spectrum and the unscaled sky continuum spectrum from the input science spectrum.

Skycorr has been designed to be instrument independent. It only requires a $1 \mathrm{D}$ science and reference sky spectrum as input. Both should be taken with the same instrumental set-up and reduced in the same way. For a good sky subtraction (especially for the unscaled continuum), both spectra should refer to a similar integrated sky area. For slit spectrographs, this depends on the slit width and the extraction in spatial direction. The former is also important for the spectral resolution. For data without flux calibration, the science and sky spectra should correspond to the same exposure time. It is expected that both files have the same format. Skycorr accepts ASCII tables, FITS tables, and 1D FITS images. The corrected output files will have the format of the input files. Several additional files are written to the output directory, which contain results of the algorithm and can be used to evaluate the quality of the sky subtraction. Skycorr has also been designed to minimise user interaction. Nevertheless, there might be code parameters that could be modified to improve the sky-subtraction results. The relevant parameters of the skycorr driver file and their default values are listed in Table 1. They are discussed in the following subsections. More details on the file structures and code parameters can be found in the skycorr user manual, which is provided along with the code.

\subsection{Line finder}

Spectral lines are identified by an approach that uses the first derivative of the spectrum (see Fig. 2). In this way, line pixels can be recognised by their steep flux gradients. Emission line peaks can be identified by a change from positive to negative values of the first derivative. The approach is insensitive to slowly varying continua. All detected lines are assembled in a line list, which is refined by an iterative method depending on the estimation of the sky line FWHM (see Sect. 2.2). This procedure requires the identification of strong, isolated lines. Therefore, the line finder checks the previously identified line peaks, applying criteria that reject spurious detections and line blends. Lines that are sufficiently separated from other lines and whose peaks have a symmetric shape are marked as isolated. The former criterion can be influenced by the user. The parameter file includes the unitless scaling parameter MIN_LINE_DIST (see Table 1) that is internally multiplied by the line FWHM in pixels, where a firstguess value is also included in the configuration file. 
Table 1. Skycorr default parameter set-up.

\begin{tabular}{|c|c|c|}
\hline Parameter $^{a}$ & Value & Short description \\
\hline VAC_AIR & $\mathrm{vac}$ & wavelengths in vacuum or air \\
\hline FWHM & 5.0 & initial estimate of line FWHM (in pixels) \\
\hline VARFWHM & 0 & $\begin{array}{l}\text { linear increase of line width with } \\
\text { wavelength? }(1=\text { yes, } 0=\text { no })\end{array}$ \\
\hline LTOL & $1 e-2$ & relative FWHM convergence criterion \\
\hline MIN_LINE_DIST & 2.5 & $\begin{array}{l}\text { minimum distance to neighbouring lines } \\
\text { for isolated lines (in FWHM) }\end{array}$ \\
\hline FLUXLIM & -1 & $\begin{array}{l}\text { minimum line peak flux (in median flux } \\
\text { of identified lines) }\end{array}$ \\
\hline FTOL & $1 e-3$ & relative $\chi^{2}$ convergence criterion \\
\hline XTOL & $1 e-3$ & relative parameter convergence criterion \\
\hline WTOL & $1 e-3$ & $\begin{array}{l}\text { convergence criterion for iterative } \\
\text { improvement of wavelength grid }\end{array}$ \\
\hline CHEBY_MAX & 7 & $\begin{array}{l}\text { maximum degree of polynomial for } \\
\text { wavelength grid correction }\end{array}$ \\
\hline CHEBY_MIN & 3 & $\begin{array}{l}\text { minimum degree of polynomial for } \\
\text { wavelength grid correction }\end{array}$ \\
\hline CHEBY_CONST & 0 . & $\begin{array}{l}\text { initial constant term for wavelength grid } \\
\text { correction }\end{array}$ \\
\hline REBINTYPE & 1 & $\begin{array}{l}\text { type of rebinning }(0=\text { simple, } \\
1=\text { asymmetric, damped sinc kernel })\end{array}$ \\
\hline WEIGHTLIM & 0.67 & $\begin{array}{l}\text { minimum relative weight of the strongest } \\
\text { line group of a pixel }\end{array}$ \\
\hline SIGLIM & 15. & $\sigma$ limit for excluding outliers \\
\hline FITLIM & 0. & $\begin{array}{l}\text { lower relative uncertainty limit for fitting } \\
\text { a line group }\end{array}$ \\
\hline
\end{tabular}

Notes. ${ }^{(a)}$ Input/output parameters are not listed.

For strong and well-separated lines, the described linefinding method is very robust. However, for low-resolution spectra with many blended lines, a large proportion of lines may be missing. Therefore, the line list of the airglow model is used (see Sect. 2.4) to find previously unidentified sky lines that have fluxes higher than the median flux of the lines identified by the derivative approach times the value of the FLUXLIM parameter specified in the parameter file. By default, FLUXLIM is set to -1 . In this case, sky lines are identified by successive iterations, starting with a threshold level of 0.005 and doubling this value in a subsequent iteration if the limit does not result in sufficient continuum pixels for the continuum interpolation (see Sect. 2.3), that is, at least $20 \%$ of all pixels distributed over more than $90 \%$ of the wavelength range. The iterative doubling of the threshold is repeated until the criterion is fulfilled or a highest value of 0.08 is reached. The number of pixels characterised as line pixels for each line included from the line list depends on the derived line FWHM (see Sect. 2.2). The combination of line pixels identified by both methods gives a good estimate of the spectral ranges covered by significant airglow lines.

\subsection{Line FWHM estimator}

To compute the airglow model (see Sect. 2.4), it is required to convert total line fluxes as provided by the input line list into fluxes per wavelength unit. Consequently, it is necessary to know the typical FWHM of the airglow lines. The line finder described in the previous Sect. 2.1 searches for isolated lines that are suitable for deriving a line width. After the subtraction of the continuum (see Sect. 2.3), the FWHM estimation can be performed by fitting a Gaussian to the line pixels belonging to each isolated line. The assumed shape of the line profile is not critical
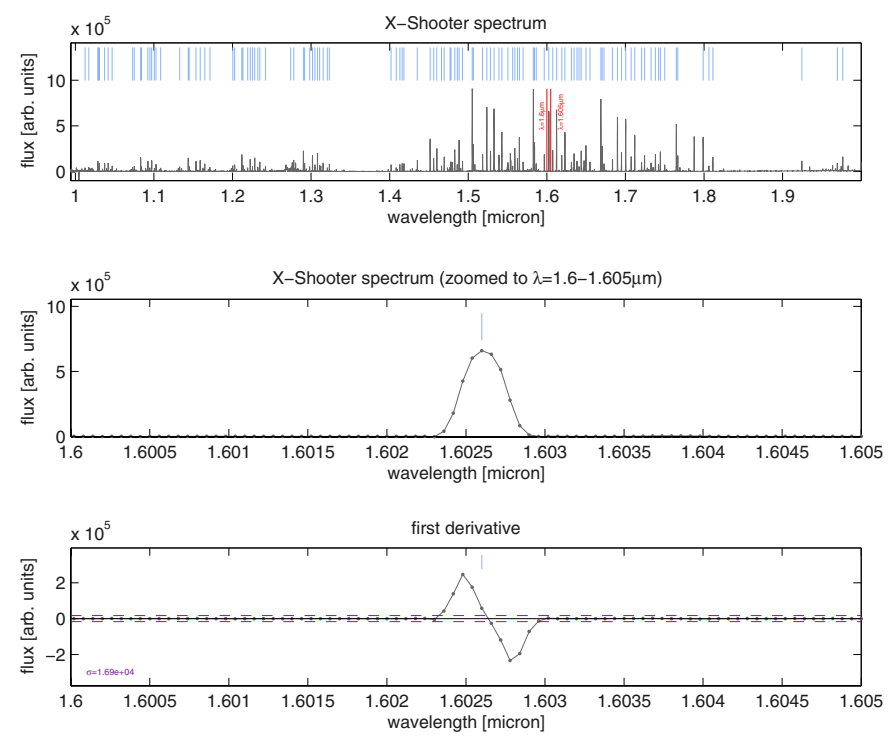

Fig. 2. Line detection in an X-shooter sky spectrum. While the upper panel shows a wide wavelength range, the middle panel focuses on a narrow range containing a prominent single emission line. In the lower panel, the first derivative of this line is given. Such patterns are used to identify emission lines. The resulting detections are marked by light blue vertical lines in the panels.

for the required accuracy. The FWHM measurements of all isolated lines are averaged to obtain the typical FWHM of the input spectrum. To avoid blended lines contributing to the resulting mean, a $\sigma$-clipping approach is applied to skip suspiciously high FWHM. If fewer than five isolated lines remain after clipping, the median FWHM is taken.

Since the FWHM estimation and the search for isolated lines (see Sect. 2.1) affect each other, an iterative approach is required, where the line finder, continuum subtractor, and FWHM estimator are called in a loop to obtain a stable and trustworthy FWHM. This iterative procedure is terminated if convergence is reached for the mean FWHM. The convergence criterion is provided by the parameter LTOL (see Table 1).

For spectrographs whose spectra show a roughly linear increase of the FWHM with wavelength and cover a wide wavelength range (see Sect. 4.1), one can set the parameter VARFWHM to 1 . In this case, the FWHM estimates of the individual lines are converted to correspond to the FWHM that would be measured at the central wavelength of the full spectrum, assuming a linear change of the FWHM with wavelength. The converted FWHM are then used to calculate the mean FWHM, as discussed above. A linear change of the FWHM is also assumed for the separation of lines and continuum (see Sect. 2.1) and the determination of the pixel contributions of the different line groups (see Sect. 2.5).

\subsection{Continuum subtraction}

In addition to spectra of astronomical objects, plain-sky spectra also show continuum emission. The main components are scattered moonlight, scattered starlight, zodiacal light, thermal emission from the lower atmosphere by greenhouse gases and the telescope itself, unresolved airglow bands, and airglow continuum emission, which is related most probably to chemical reactions in the upper atmosphere involving nitric oxide (Khomich et al. 2008 and references therein; Noll et al. 2012). In addition, an instrument-related continuum due to internal scattering and similar effects could significantly contribute (see e.g. Ellis \& Bland-Hawthorn 2008; Vernet et al. 2011). As Fig. 3 indicates, 


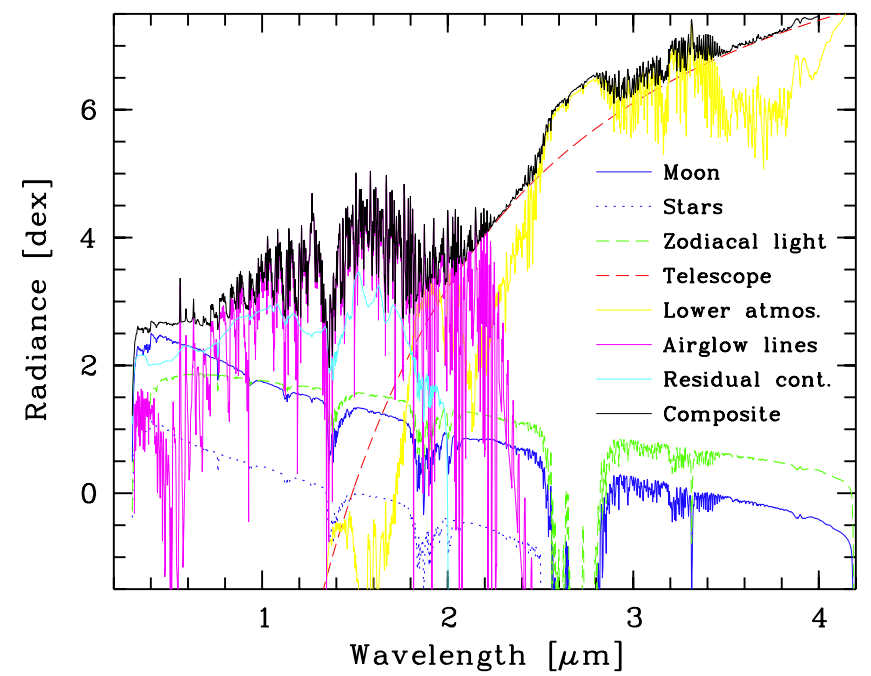

Fig. 3. Components of the Cerro Paranal sky model for wavelengths between 0.3 and $6 \mu \mathrm{m}$ in logarithmic radiance units. The example with the Moon above the horizon shows the scattered moonlight, scattered starlight, zodiacal light, thermal emission by telescope and instrument, molecular emission of the lower atmosphere, airglow emission lines of the upper atmosphere, and airglow/residual continuum. The (optical) model components are described in Noll et al. (2012) except for the improved scattered moonlight model, which is discussed in Jones et al. (2013).

the main continuum component is the airglow/residual contin$\mathrm{uum}^{3}$, which dominates shortwards of the thermal regime with the exception of the ultraviolet (UV) and optical if the Moon is up.

The variable airglow continuum (see Noll et al. 2012) and the thermal continuum, which can significantly change by temperature differences of a few degrees, cannot be corrected for by a fitting procedure as is done for the airglow lines (see Sect. 2.4), since object and sky continuum cannot be separated in the science spectrum (cf. Sect. 1). Hence, skycorr performs a simple continuum subtraction without scaling. To achieve reliable results, this approach requires that the object continuum is distinctly brighter than the variation in the sky continuum (see Sect. 3.2 for more details). Note that the strong thermal methane and water vapour lines of the lower atmosphere at wavelengths beyond $2.3 \mu \mathrm{m}$ are also handled as continuum. This can lead to significant sky-subtraction residuals for inaccurate wavelength solutions (see Sect. 2.6).

Skycorr obtains the continua in the input science and sky spectra using line identification flags set in the course of the line search described in Sect. 2.1. All pixels not flagged as line pixels are connected by linear interpolation. If the identification of continuum pixels is reliable, this is the most efficient approach even for line blends that cover wide wavelength ranges.

\subsection{Airglow model}

The wavelength range from the near-UV to the near-IR is characterised by strong emission lines. Most of them constitute band structures. This airglow (see Khomich et al. (2008) for a comprehensive discussion) mostly originates in the mesopause

\footnotetext{
3 Note that the airglow continuum is very difficult to determine. Its measured intensity strongly depends on the accuracy of the other components, the quality of the flux calibration, and possible instrumental continua. For this reason, this model component should also be seen as residual continuum.
}

region at about $90 \mathrm{~km}$. In addition, some lines arise in the ionospheric F2-layer at about $270 \mathrm{~km}$. In general, airglow is caused by chemiluminescence, that is, by chemical reactions that lead to light emission by the decay of excited states of reaction products. Apart from atomic oxygen and sodium, the oxygen $\left(\mathrm{O}_{2}\right)$ and hydroxyl $(\mathrm{OH})$ molecules are the most important reaction products in this context. In general, airglow lines show strong variability from time scales in the order of minutes to decades. This behaviour can be explained by the solar activity cycle, seasonal changes in temperature, pressure, and chemical composition of the emission layers, the day-night contrast, dynamical effects such as planetary and gravity waves, or geomagnetic disturbances. The dynamical effects also cause spatial intensity variations. In addition, the airglow intensity depends on the projected emission layer width, which is a function of the zenith distance (van Rhijn 1921; Noll et al. 2012).

Because the airglow is highly variable, the strength of the emission lines in a reference sky spectrum for sky correction has to be adapted. This is achieved by a fitting procedure that is discussed in Sect. 2.5. Since object emission lines should not be reproduced by the optimised sky spectrum and finally removed, it is advisable to scale as many lines as possible by a single fitting parameter. Every group should contain airglow lines that are not affected by object lines and that can be used to determine a realistic correction factor for the reference sky spectrum. The number of lines that can be combined is limited by the fact that they should show an almost identical variability behaviour.

As a basis for the definition of suitable line groups, the airglow line model developed for the Cerro Paranal sky-radiance model (Noll et al. 2012) was used. This semi-empirical model consists of a line list with line intensities for mean observing conditions and prescriptions for the correction of the line strength depending on molecular species, solar activity, season, time of night, and zenith distance of the target. The latter three input parameters can be retrieved from the FITS header of the sky spectrum file. The solar activity is traced by the solar radio flux at $10.7 \mathrm{~cm}$, which can be provided either by the parameter SOLFLUX in the configuration file or by the corresponding monthly average (default) in a file offered by http: //www . spaceweather.gc . ca. In the wavelength range from 0.3143 to $0.9228 \mu \mathrm{m}$, the line list consists of data taken from Cosby et al. (2006). They incorporated the emission line atlas of Hanuschik (2003) based on observations with the VLT high-resolution echelle spectrograph UVES (Dekker et al. 2000). To fill a gap at about $0.86 \mu \mathrm{m}$, this list was supplemented by unpublished UVES data of R. Hanuschik. At longer wavelengths, the calculated $\mathrm{OH}$ lines of Rousselot et al. (2000) were included. However, their line strengths were corrected for the Einstein factors of Goldman et al. (1998) instead of using the original ones of Mies (1974). This resulted in correction factors for $\mathrm{OH}$ band strengths between 0.38 and 2.06. Moreover, the flux decrease of airglow lines by molecular absorption in the lower atmosphere was corrected. To this end, Gaussian profiles for each airglow line with Doppler line widths for typical temperatures of about $200 \mathrm{~K}$ were convolved with the high-resolution $\left(\lambda / \Delta \lambda \approx 10^{6}\right)$ Cerro Paranal annual-mean transmission curve for an airmass of $1.25^{4}$

4 Although the atmospheric transmission depends on airmass and weather conditions, only a fixed airglow flux correction was applied to avoid time-consuming calculations at very high resolution and the input of temperature and water vapour profiles. Moreover, the optical airglow atlas of Hanuschik (2003) is also characterised by a fixed transmission correction because the UVES mean spectra were used. For most observing conditions, the deviation of the true airglow absorption from the assumed one is expected to be minor in terms of the results of skycorr. 
(see Noll et al. 2012). The transmission curve was computed by means of the radiative transfer code LBLRTM (see Clough et al. 2005). Note that skycorr applies the Cerro Paranal mean transmission curve for zenith (corrected for the target airmass) to the unextincted fluxes in the input line list. For this purpose, the line list contains separate columns for unextincted line fluxes and zenithal transmission values. Finally, the Rousselot et al. lines were scaled to the Cosby et al. lines between 0.642 and $0.858 \mu \mathrm{m}^{5}$. The strongest $\mathrm{O}_{2}$ bands in the near-IR at 1.27 and $1.58 \mu \mathrm{m}$ were included in the line list by adding data from the HITRAN molecular line database (see Rothman et al. 2009). The mean band strength was roughly estimated by evaluating the ratio of $\mathrm{O}_{2}$ to $\mathrm{OH}$ lines in $26 \mathrm{NIR}$-arm X-shooter spectra (see Vernet et al. 2011 for details on the instrument; see also Sect. 3). For this purpose, the $\mathrm{O}_{2}$ lines had to be extincted depending on the airmass values of the $\mathrm{X}$-shooter spectra. For the $1.27 \mu \mathrm{m}$ band, this caused significant changes in the line fluxes because of the strong resonant absorption of airglow photons by tropospheric/stratospheric $\mathrm{O}_{2}$ molecules in the ground state.

The Cerro Paranal sky model assigns the listed lines to the five different variability classes (1) green O I, (2) Na ID, (3) red $\mathrm{O} \mathrm{I}$, (4) $\mathrm{OH}$, and (5) $\mathrm{O}_{2}$ (see Noll et al. 2012). These variability classes result from analysing a sample of 1189 optical FORS1 spectra (Patat 2008). From this sample, the lines' dependence on solar radio flux and time of observation was derived. The latter was quantified using a grid of six two-month periods starting with Dec./Jan. and three night-time bins of equal length. The reference line strengths in the line list represent the mean of the five solar activity cycles 19 to 23, that is, the years 1954 to 2007 . The ratios of line strengths of different variability classes can easily vary by a factor of two and more.

Assigning airglow lines to the five classes using the rough predictions from the sky model is not sufficient for achieving a line intensity accuracy on the per cent level, which is required for a sky-subtraction procedure like skycorr. Typically, intensity variations of lines within a variability class are stronger than can be tolerated.

In principle, an identical variability behaviour can be expected for transitions with the same upper state. In this case, the ratios of line intensities should be fixed and only determined by quantities such as Einstein coefficients and statistical weights. On the other hand, the excitation and population of different energy levels depends on variable quantities such as temperature and chemical abundances. Therefore, it is promising to define line groups depending on the upper energy level. However, taking all relevant states of the molecules $\mathrm{OH}$ and $\mathrm{O}_{2}$ into account would result in very many line groups. Moreover, each group would consist of only a few significant lines. This would result in statistical fluctuations, which could make the line intensity correction uncertain if crucial lines of a group were affected for example by detector defects or object emission lines (see Sect. 2.5). Fortunately, as their energies are very different, it is possible to separate electronic, vibrational, and rotational transitions of molecules. The electronic/vibrational transition determines the band and the rotational transition identifies a single line or doublet (as in case of $\mathrm{OH}$ ) within a band. Since the distribution of energy levels is very similar for all bands of an electronic transition, each line can be assigned to two different classes that are defined by the upper vibrational and rotational

\footnotetext{
5 The calculated OH line spectrum of Rousselot et al. (2000) covers a wider wavelength range $(0.614-2.624 \mu \mathrm{m})$ than the published atlas related to observations with the VLT spectrograph ISAAC (0.997-2.252 $\mu \mathrm{m})$.
}

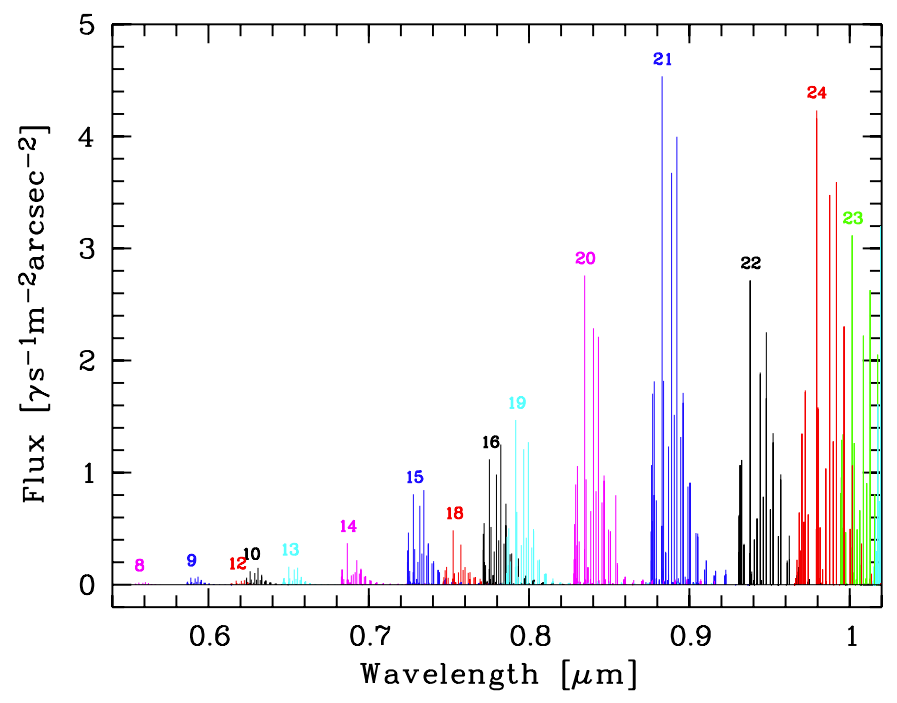

Fig. 4. A-group identifications of $\mathrm{OH}$ bands (cf. Table 2) in the wavelength range between 0.54 and $1.02 \mu \mathrm{m}$ that have Q1(1.5) lines (see Table 3 and Fig. 6) stronger than $0.01 \gamma^{-1} \mathrm{~m}^{-2} \operatorname{arcsec}^{-2}$. The wavelengths and zenithal mean fluxes (considering absorption in the lower atmosphere) tabulated in the input line list are plotted. Note that the bands with numbers up to 21 appear twice as strong as the bands at longer wavelengths, since the corresponding lines were taken from the Hanuschik (2003) atlas, where $\mathrm{OH}$ doublets are often unresolved and are listed as only one line.

state. This approach reduces the number of required line groups significantly. Moreover, for $\mathrm{OH}$ only the electronic ground state is relevant, which splits up into the sub-levels $X^{2} \Pi_{1 / 2}$ and $X^{2} \Pi_{3 / 2}$ because of the coupling of spin and orbital angular momentum (see Rousselot et al. 2000). For $\mathrm{O}_{2}$, the electronic transitions are more important than the vibrational ones, since the intensity differences of the bands of an electronic transition are very large. Consequently, there are only three $\mathrm{O}_{2}$ bands that significantly contribute to the airglow, namely $\mathrm{O}_{2}(\mathrm{~b}-\mathrm{X})(0-1)^{6}$ (the very strong $(0-0)$ band is almost completely absorbed in the lower atmosphere), $\mathrm{O}_{2}(\mathrm{a}-\mathrm{X})(0-0)$, and $\mathrm{O}_{2}(\mathrm{a}-\mathrm{X})(0-1)$ (see Khomich et al. 2008). The $\mathrm{O}_{2}$ bands at near-UV and blue wavelengths are weak and can only be resolved at very high resolution. For most instruments, these bands therefore appear as a pseudo-continuum.

Tables 2 and 3 list the final grouping of airglow lines. Line groups with the same upper electronic/vibrational level are called $A$ groups and those with the same $(\mathrm{OH})$ or a similar $\left(\mathrm{O}_{2}\right)$ upper rotational level are labelled $B$ groups. Most $\mathrm{OH}$ bands (apart from a few very weak ones) are identified in Figs. 4 and 5. Although bands such as $\mathrm{OH}(4-1)$ and $\mathrm{OH}(4-2)$ have the same upper vibrational level, they represent independent variability groups. Since real data suffer from calibration uncertainties, this procedure is necessary, even though the number of $A$ groups increases significantly. Because $\mathrm{OH}$ bands with the same upper vibrational level are widely separated, it is safer to vary these bands independently. Figures 6-9 show identifications of the rotational $B$ groups for $\mathrm{OH}(4-2)$ as an example of an $\mathrm{OH}$ band, $\mathrm{O}_{2}(\mathrm{~b}-\mathrm{X})(0-1), \mathrm{O}_{2}(\mathrm{a}-\mathrm{X})(0-0)$, and $\mathrm{O}_{2}(\mathrm{a}-\mathrm{X})(0-1)$, respectively. Although the two $\mathrm{O}_{2}(\mathrm{a}-\mathrm{X})$ bands belong to the same roto-vibrational system, their $B$ groups were defined separately because of the completely different line flux distribution. This

\footnotetext{
6 The notation used is as follows: molecule (upper - lower electronic state) (upper - lower vibrational state). The letters a, b, and $\mathrm{X}$ are shortcuts for the states $a^{1} \Delta_{g}, b^{1} \Sigma_{g}^{+}$, and $X^{3} \Sigma_{g}^{-}$. The vibrational states are numbered depending on the energy and starting from 0 for the lowest level.
} 
Table 2. Description of $A$ groups in the input line list.

\begin{tabular}{|c|c|c|c|}
\hline ID & $N_{\text {lin }}$ & $\begin{array}{c}\lambda \text { range } \\
{[\mu \mathrm{m}]}\end{array}$ & Description \\
\hline 1 & 61 & $0.314-0.872$ & $\begin{array}{l}\text { green O I at } 557.7 \mathrm{~nm} \\
+ \text { unidentified lines }\end{array}$ \\
\hline 2 & 3 & $0.589-0.770$ & $\begin{array}{l}\mathrm{NaID}+\text { other lines } \\
\text { from alkali metals }\end{array}$ \\
\hline 3 & 23 & $0.389-0.845$ & $\begin{array}{l}\text { red O I at } 630.0 \mathrm{~nm} \\
+ \text { other thermospheric lines }\end{array}$ \\
\hline 4 & 1 & $0.467-0.467$ & $\mathrm{OH}(7-0)$ \\
\hline 5 & 8 & $0.491-0.495$ & $\mathrm{OH}(8-1)$ \\
\hline 6 & 22 & $0.519-0.536$ & $\mathrm{OH}(9-2)$ \\
\hline 7 & 12 & $0.526-0.535$ & $\mathrm{OH}(6-0)$ \\
\hline 8 & 23 & $0.554-0.570$ & $\mathrm{OH}(7-1)$ \\
\hline 9 & 41 & $0.587-0.634$ & $\mathrm{OH}(8-2)$ \\
\hline 10 & 49 & $0.624-0.655$ & $\mathrm{OH}(9-3)$ \\
\hline 11 & 2 & $0.672-0.674$ & $\mathrm{OH}(10-4)$ \\
\hline 12 & 27 & $0.614-0.695$ & $\mathrm{OH}(5-0)$ \\
\hline 13 & 83 & $0.647-0.754$ & $\mathrm{OH}(6-1)$ \\
\hline 14 & 113 & $0.681-0.782$ & $\mathrm{OH}(7-2)$ \\
\hline 15 & 111 & $0.720-0.815$ & $\mathrm{OH}(8-3)$ \\
\hline 16 & 72 & $0.768-0.822$ & $\mathrm{OH}(9-4)$ \\
\hline 17 & 7 & $0.827-0.839$ & $\mathrm{OH}(10-5)$ \\
\hline 18 & 85 & $0.745-0.910$ & $\mathrm{OH}(4-0)$ \\
\hline 19 & 113 & $0.781-0.914$ & $\mathrm{OH}(5-1)$ \\
\hline 20 & 111 & $0.826-0.916$ & $\mathrm{OH}(6-2)$ \\
\hline 21 & 110 & $0.873-0.937$ & $\mathrm{OH}(7-3)$ \\
\hline 22 & 116 & $0.931-1.007$ & $\mathrm{OH}(8-4)$ \\
\hline 23 & 120 & $0.994-1.081$ & $\mathrm{OH}(9-5)$ \\
\hline 24 & 100 & $0.965-1.043$ & $\mathrm{OH}(3-0)$ \\
\hline 25 & 110 & $1.015-1.098$ & $\mathrm{OH}(4-1)$ \\
\hline 26 & 112 & $1.069-1.168$ & $\mathrm{OH}(5-2)$ \\
\hline 27 & 118 & $1.129-1.236$ & $\mathrm{OH}(6-3)$ \\
\hline 28 & 120 & $1.197-1.314$ & $\mathrm{OH}(7-4)$ \\
\hline 29 & 124 & $1.275-1.420$ & $\mathrm{OH}(8-5)$ \\
\hline 30 & 128 & $1.366-1.531$ & $\mathrm{OH}(9-6)$ \\
\hline 31 & 112 & $1.392-1.558$ & $\mathrm{OH}(2-0)$ \\
\hline 32 & 118 & $1.461-1.654$ & $\mathrm{OH}(3-1)$ \\
\hline 33 & 122 & $1.537-1.743$ & $\mathrm{OH}(4-2)$ \\
\hline 34 & 122 & $1.622-1.842$ & $\mathrm{OH}(5-3)$ \\
\hline 35 & 124 & $1.717-1.978$ & $\mathrm{OH}(6-4)$ \\
\hline 36 & 126 & $1.825-2.110$ & $\mathrm{OH}(7-5)$ \\
\hline 37 & 130 & $1.951-2.265$ & $\mathrm{OH}(8-6)$ \\
\hline 38 & 130 & $2.101-2.454$ & $\mathrm{OH}(9-7)$ \\
\hline 39 & 450 & $0.314-0.532$ & $\mathrm{O}_{2}(\mathrm{~A}-\mathrm{X})($ Herzberg I) \\
\hline 40 & 5 & $0.324-0.410$ & $\mathrm{O}_{2}(\mathrm{c}-\mathrm{X})($ Herzberg II) \\
\hline 41 & 396 & $0.326-0.550$ & $\mathrm{O}_{2}\left(\mathrm{~A}^{\prime}-\mathrm{a}\right)$ (Chamberlain) \\
\hline 42 & 65 & $0.382-0.509$ & $\mathrm{O}_{2}(\mathrm{c}-\mathrm{b})$ \\
\hline 43 & 208 & $0.656-0.806$ & $\mathrm{O}_{2}(\mathrm{~b}-\mathrm{X})\left(v^{\prime}>v^{\prime \prime}\right)$ \\
\hline 44 & 194 & $0.761-0.816$ & $\mathrm{O}_{2}(\mathrm{~b}-\mathrm{X})\left(v^{\prime}=v^{\prime \prime}\right)$ \\
\hline 45 & 103 & $0.861-0.922$ & $\begin{array}{l}\mathrm{O}_{2}(\mathrm{~b}-\mathrm{X})\left(v^{\prime}<v^{\prime \prime}\right. \\
\text { including atm. } 0-1 \text { band })\end{array}$ \\
\hline 46 & 161 & $1.240-1.305$ & $\begin{array}{l}\mathrm{O}_{2}(\mathrm{a}-\mathrm{X})(0-0) \\
\text { (IR atmospheric system) }\end{array}$ \\
\hline 47 & 73 & $1.555-1.598$ & $\begin{array}{l}\mathrm{O}_{2}(\mathrm{a}-\mathrm{X})(0-1) \\
(\mathrm{IR} \text { atmospheric system) }\end{array}$ \\
\hline
\end{tabular}

is caused by self-absorption in the $(0-0)$ band. $B$ groups of $\mathrm{O}_{2}$ bands consist of lines from two rotational upper levels to ensure that enough lines can be identified for the group scaling (see Sect. 2.5). The weak lines of each band are not included in a $B$ group, since they are difficult to fit. Furthermore, this measure avoids a degeneration of fit parameters.

The described grouping is more complex than that of Davies (2007), since Davies only incorporated near-IR OH bands,

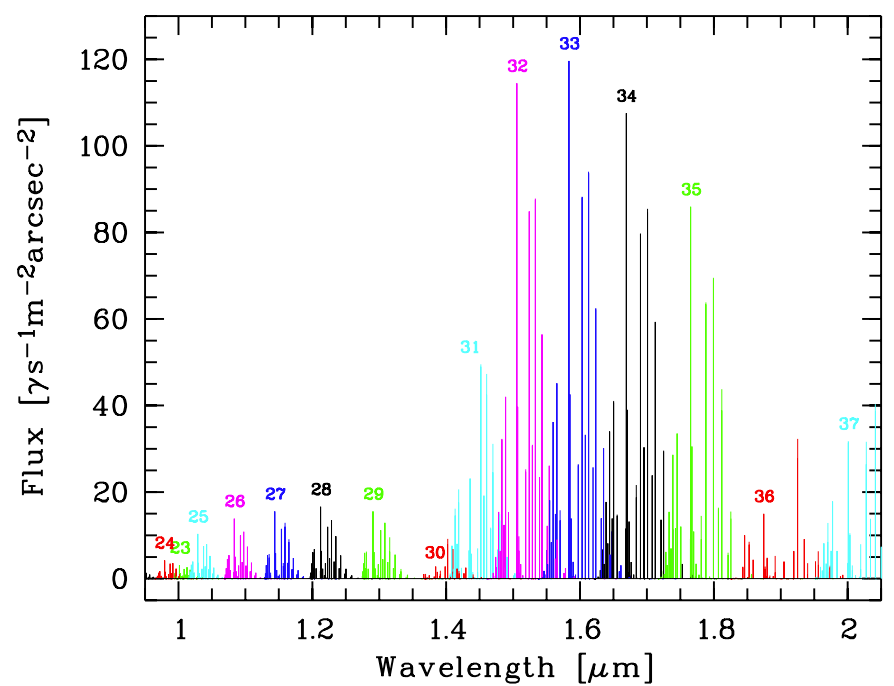

Fig. 5. A-group identifications of the $\mathrm{OH}$ bands (cf. Table 2) in the wavelength range between 0.95 and $2.05 \mu \mathrm{m}$. The wavelengths and zenithal mean fluxes tabulated in the input line list are plotted.

Table 3. Description of $B$ groups in the input line list.

\begin{tabular}{ccll}
\hline \hline ID & Molecule & Upper state(s) & Remarks $^{a}$ \\
\hline 1 & $\mathrm{OH}$ & $X^{2} \Pi_{1 / 2}, J^{\prime}=1 / 2$ & $\mathrm{Q} 2(0.5), \mathrm{P} 2(1.5)$ \\
2 & $\mathrm{OH}$ & $X^{2} \Pi_{3 / 2}, J^{\prime}=3 / 2$ & $\mathrm{Q} 1(1.5), \mathrm{P} 1(2.5)$ \\
3 & $\mathrm{OH}$ & $X^{2} \Pi_{1 / 2}, J^{\prime}=3 / 2$ & $\mathrm{R} 2(0.5), \mathrm{Q} 2(1.5), \mathrm{P} 2(2.5)$ \\
4 & $\mathrm{OH}$ & $X^{2} \Pi_{3 / 2}, J^{\prime}=5 / 2$ & $\mathrm{R} 1(1.5), \mathrm{Q} 1(2.5), \mathrm{P} 1(3.5)$ \\
5 & $\mathrm{OH}$ & $X^{2} \Pi_{1 / 2}, J^{\prime}=5 / 2$ & $\mathrm{R} 2(1.5), \mathrm{Q} 2(2.5), \mathrm{P} 2(3.5)$ \\
6 & $\mathrm{OH}$ & $X^{2} \Pi_{3 / 2}, J^{\prime}=7 / 2$ & $\mathrm{R} 1(2.5), \mathrm{Q} 1(3.5), \mathrm{P} 1(4.5)$ \\
7 & $\mathrm{OH}$ & $X^{2} \Pi_{1 / 2}, J^{\prime}=7 / 2$ & $\mathrm{R} 2(2.5), \mathrm{Q} 2(3.5), \mathrm{P} 2(4.5)$ \\
8 & $\mathrm{OH}$ & $X^{2} \Pi_{3 / 2}, J^{\prime}=9 / 2$ & $\mathrm{R} 1(3.5), \mathrm{Q} 1(4.5), \mathrm{P} 1(5.5)$ \\
9 & $\mathrm{OH}$ & $X^{2} \Pi_{1 / 2}, J^{\prime}=9 / 2$ & $\mathrm{R} 2(3.5), \mathrm{Q} 2(4.5), \mathrm{P} 2(5.5)$ \\
10 & $\mathrm{OH}$ & $X^{2} \Pi_{3 / 2}, J^{\prime}=11 / 2$ & $\mathrm{R} 1(4.5), \mathrm{Q} 1(5.5), \mathrm{P} 1(6.5)$ \\
11 & $\mathrm{O}_{2}$ & $b^{1} \Sigma_{g}^{+}, J^{\prime}=0,2,4$ & \\
12 & $\mathrm{O}_{2}$ & $b^{1} \Sigma_{g}^{+}, J^{\prime}=6,8$ & \\
13 & $\mathrm{O}_{2}$ & $b^{1} \Sigma_{g}^{+}, J^{\prime}=10,12$ & \\
14 & $\mathrm{O}_{2}$ & $b^{1} \Sigma_{g}^{+}, J^{\prime}=14,16$ & \\
15 & $\mathrm{O}_{2}$ & $a^{1} \Delta_{g}, J^{\prime}=2,4$ & $v^{\prime \prime}=0$ \\
16 & $\mathrm{O}_{2}$ & $a^{1} \Delta_{g}, J^{\prime}=6,8$ & $v^{\prime \prime}=0$ \\
17 & $\mathrm{O}_{2}$ & $a^{1} \Delta_{g}, J^{\prime}=10,12$ & $v^{\prime \prime}=0$ \\
18 & $\mathrm{O}_{2}$ & $a^{1} \Delta_{g}, J^{\prime}=14,16$ & $v^{\prime \prime}=0$ \\
19 & $\mathrm{O}_{2}$ & $a^{1} \Delta_{g}, J^{\prime}=18,20$ & $v^{\prime \prime}=0$ \\
20 & $\mathrm{O}_{2}$ & $a^{1} \Delta_{g}, J^{\prime}=20,22$ & $v^{\prime \prime}=0$ \\
21 & $\mathrm{O}_{2}$ & $a^{1} \Delta_{g}, J^{\prime}=2,4$ & $v^{\prime \prime} \neq 0$ \\
22 & $\mathrm{O}_{2}$ & $a^{1} \Delta_{g}, J^{\prime}=6,8$ & $v^{\prime \prime} \neq 0$ \\
23 & $\mathrm{O}_{2}$ & $a^{1} \Delta_{g}, J^{\prime}=10,12$ & $v^{\prime \prime} \neq 0$ \\
24 & $\mathrm{O}_{2}$ & $a^{1} \Delta_{g}, J^{\prime}=14,16$ & $v^{\prime \prime} \neq 0$ \\
\hline
\end{tabular}

Notes. ${ }^{(a)} \mathrm{OH}$ rotational transitions (identified by the branch and total angular momentum of the lower state $J^{\prime \prime}$ ) or lower vibrational level $v^{\prime \prime}$ for $\mathrm{O}_{2}(\mathrm{a}-\mathrm{X})$ transitions.

$\mathrm{O}_{2}(\mathrm{a}-\mathrm{X})(0-0)$, and two rotational groups resembling our $B 2$ and $B 4$ classes (see Table 3 ).

\subsection{Airglow line fitter}

To prepare a reference sky line spectrum for the sky subtraction in a science spectrum, the line fluxes have to be adapted. To this end, Davies (2007) divided the wavelength range into sections depending on the $\mathrm{OH}$ band structure and subsequently scaled 
S. Noll et al.: Skycorr: A general tool for spectroscopic sky subtraction

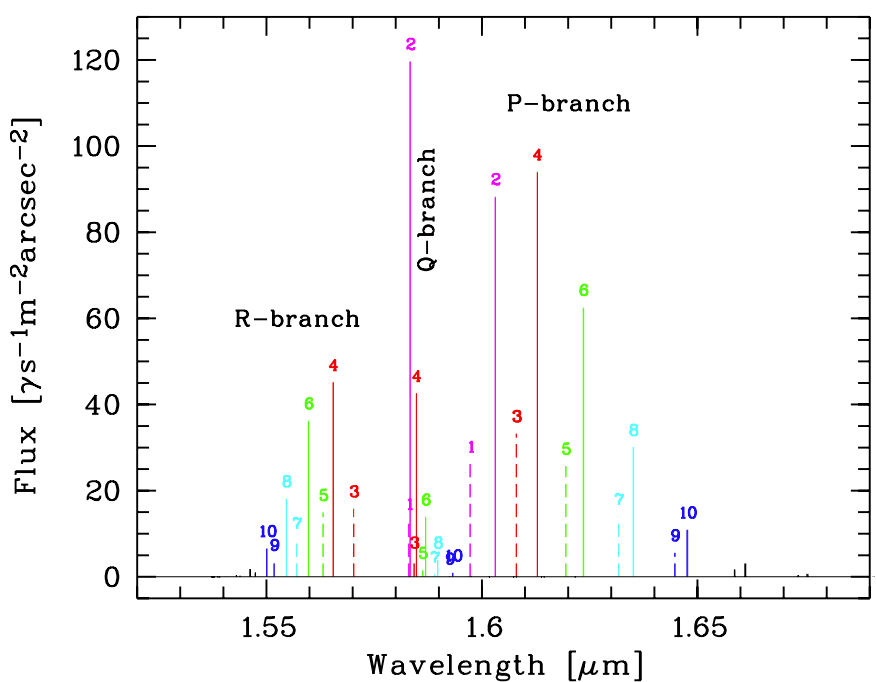

Fig. 6. $B$-group identifications of the transitions of an $\mathrm{OH}$ band with the same rotational upper state (cf. Table 3 ). The tabulated wavelengths and zenithal mean fluxes of the lines of the $\mathrm{OH}(4-2)$ band are shown as example. Dashed and solid lines indicate transitions of the $X^{2} \Pi_{1 / 2}$ and $X^{2} \Pi_{3 / 2}$ state. The figure also indicates the R-, Q-, and P-branches that correspond to transitions with a change of the total angular momentum by $-1,0$, and 1 , respectively.

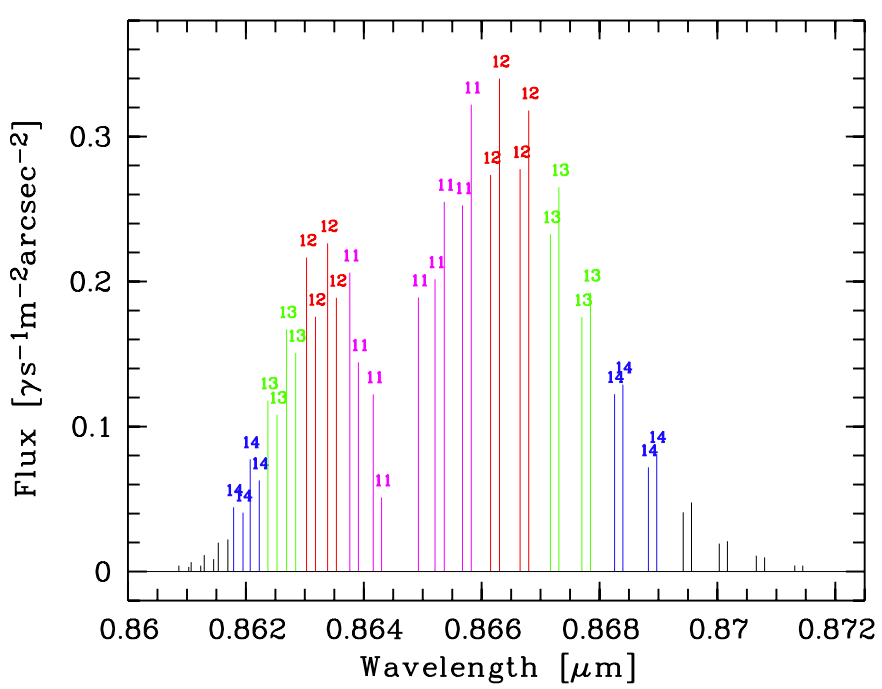

Fig. 7. $B$-group identifications of the transitions of the band $\mathrm{O}_{2}(\mathrm{~b}-\mathrm{X})(0-1)$ with a similar rotational upper state (cf. Table 3$)$. The tabulated wavelengths and zenithal mean fluxes of the lines of the 4 different branches (2 R- and 2 P-branches) are shown (cf. Fig. 6).

these segments independently according to the sections' flux ratio of the science and sky spectra. This can be problematic where different line groups have significant overlap. While $\mathrm{OH}$ bands in $H$-band spectra do not significantly overlap and are not blended with strong bands of other molecules, at lower wavelengths the situation is less favourable (see Sect. 2.4). Even so, it is difficult to measure the scaling factors for groups with the same upper rotational level. Here, the flux of individual lines has to be derived, which requires that the selected lines are isolated. Typically, this is not the case for $\mathrm{Q}$ transitions, which are characterised by a constant total angular momentum (see Fig. 6). Furthermore, the separation of variability groups becomes even more difficult if the spectral resolution is relatively low.

To overcome these limitations, skycorr uses a completely different approach to obtain the scaling factors for the different

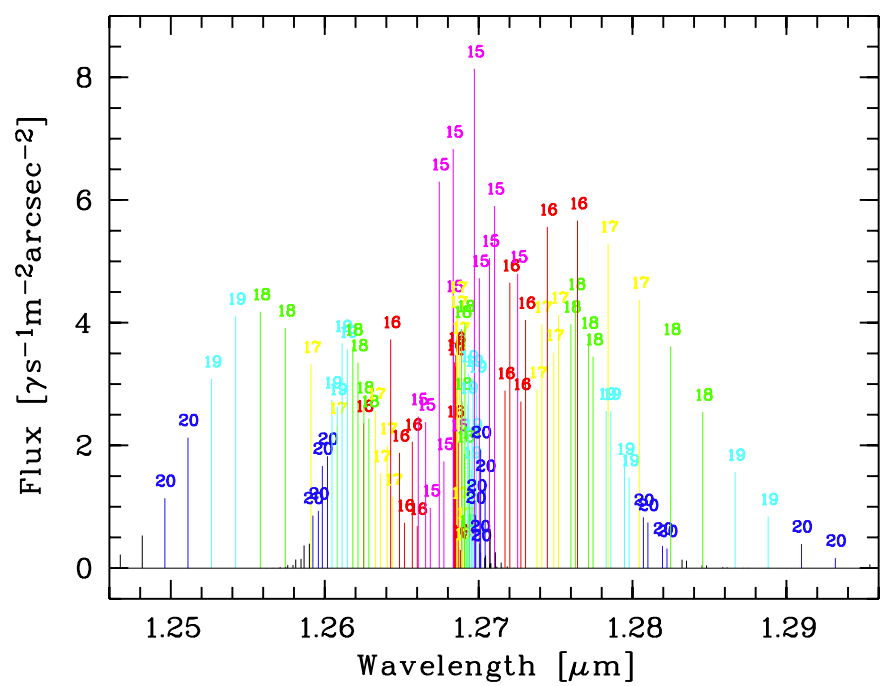

Fig. 8. $B$-group identifications of the transitions of the band $\mathrm{O}_{2}(\mathrm{a}-\mathrm{X})(0-0)$ with a similar rotational upper state (cf. Table 3$)$. The tabulated wavelengths and zenithal mean fluxes of the lines of the 9 different branches (3 R-, 3 Q-, and 3 P-branches) are shown (cf. Fig. 6). The band is strongly affected by self-absorption in the lower atmosphere.

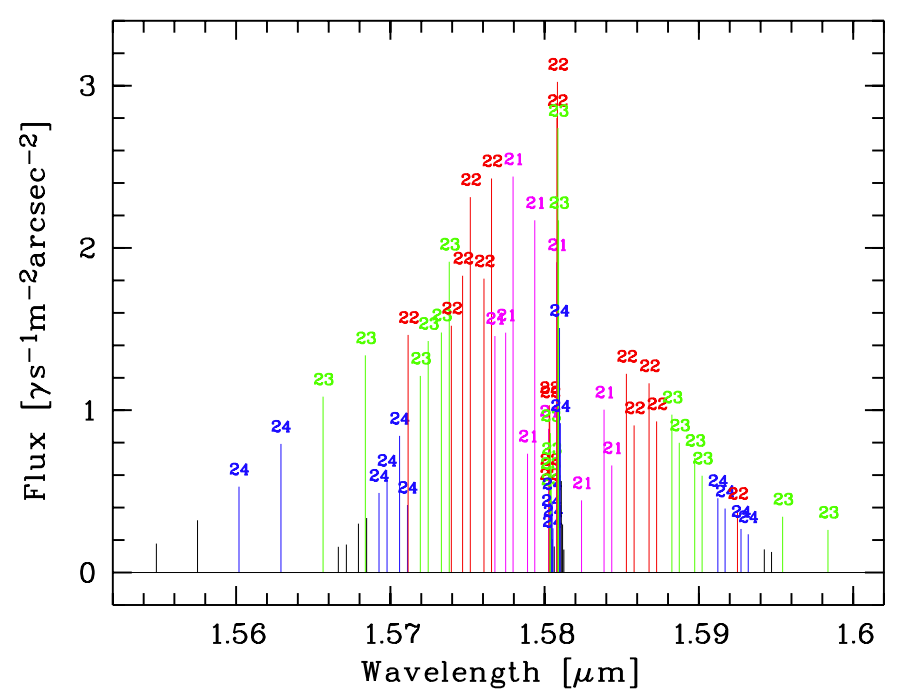

Fig. 9. $B$-group identifications of the transitions of the band $\mathrm{O}_{2}(\mathrm{a}-\mathrm{X})(0-1)$ with a similar rotational upper state (cf. Table 3$)$. The tabulated wavelengths and zenithal mean fluxes of the lines of the 9 different branches (3 R-, 3 Q-, and 3 P-branches) are shown (cf. Fig. 6).

line groups defined in Sect. 2.4. In skycorr, the contributions of the line groups to each pixel of the sky spectrum are estimated. Subsequently, the resulting spectra for each line class are scaled.

This is performed by applying the airglow model presented in the previous section. The wavelengths and intensities of the lines and their group identifications can be converted into intensities of the different line groups for each pixel. This requires a convolution of the lines from the line list with a kernel similar to the instrumental profile of the observed spectra. The mean FWHM of the sky lines, which was obtained in a previous step (see Sect. 2.2), is used to create a sufficiently realistic Gaussian kernel. To treat intensity ratios of overlapping lines as realistically as possible, the airglow variability model from Noll et al. (2012) (see Sect. 2.4) was included. This allows one to roughly correct for the influence of solar activity, season, and time of night on the main line variability classes green $\mathrm{OI}, \mathrm{NaID}$, red $\mathrm{O}$, $\mathrm{OH}$, and $\mathrm{O}_{2}$. 
When different line groups contribute to the same pixel, the sky scaling factors cannot be derived by a simple division of line fluxes of science and sky spectrum anymore. Instead, each scaling factor of the individual line groups has to be included in a fitting procedure as a free fitting parameter. For this purpose, the $\mathrm{C}$ version of the least-squares fitting library MPFIT by C. Markwardt ${ }^{7}$ based on the FORTRAN fitting routine MINPACK-1 by Moré et al. (1980) is used. The $\chi^{2}$ minimisation procedure of this routine is based on a LevenbergMarquardt technique, an iterative search algorithm characterised by gradient-controlled jumps in parameter space. Since this technique is potentially prone to find local minima, reasonable start values and constraints on the fit parameters are required. For this reason, the mean ratios of the line peaks in the science and sky spectrum are determined for each line group (see Sect. 2.4). Only the spectrum pixels are included that were identified as line peaks (see Sect. 2.1) or are separated from peaks by not more than half the line FWHM (see Sect. 2.2) and have a relative contribution of the selected line group of at least WEIGHTLIM (default: 0.67; see Table 1). Moreover, pixels with unreasonable flux ratios are rejected by applying a global $\sigma$ limit that is derived from the full set of line peaks and is provided by the parameter SIGLIM (default: 15; see Table 1). In this way, strong object emission lines can be identified in the science line spectrum and excluded. Finally, the $\sigma$-clipping approach with variable $\sigma$ limit described in Sect. 2.2 is applied to the selected pixels of each group separately to further improve the pixel selection. The remaining pixels of this procedure are taken for the initial line group scaling and fitting algorithm, meaning that only these pixels are considered for the $\chi^{2}$ calculation. If suitable pixels cannot be found for a line group, a mean flux ratio of the corresponding system of roto-vibrational bands (e.g. $\mathrm{OH}$; see Sect. 2.4) or a global flux ratio is taken for $A$ groups and a value of 1 is assumed for $B$ groups. For most sky spectra, this approach is expected to result in a good first guess sufficient for achieving a rapid convergence to the global minimum.

As an option, the fitting can be restricted to uncertain line groups alone. The decision on the group selection depends on the parameter FITLIM (see Table 1), which provides a limiting ratio of the root mean square (rms) and the mean of the groupspecific scaling factors. By default, this value is set to 0 , meaning that all fittable line groups are considered.

\subsection{Correction of wavelength grid}

Since the sky lines of the science spectrum are removed by a scaled reference sky line spectrum, it is imperative that the wavelength grids of both spectra are aligned. Differences of less than a pixel can already significantly deteriorate the quality of the sky subtraction. Relatively large deviations can occur if a lamp spectrum taken in daytime at different ambient conditions as the science spectrum is used for the wavelength calibration. However, even subpixel shifts that are routinely observed in data taken under perfect conditions can cause problems.

For this reason, skycorr offers an optional correction of the wavelength grid by applying a Chebyshev polynomial of degree $n_{\mathrm{w}}$

$$
\lambda^{\prime}=\sum_{i=0}^{n_{\mathrm{w}}} c_{i} t_{i}
$$

\footnotetext{
http://www.physics.wisc.edu/ craigm/idl/cmpfit.html
}

$$
\begin{aligned}
& \text { where } \\
& t_{i}= \begin{cases}1 & \text { for } i=0 \\
\lambda & \text { for } i=1 \\
2 \lambda t_{i-1}-t_{i-2} & \text { for } i \geq 2\end{cases}
\end{aligned}
$$

and $\lambda$ ranging from -1 to 1 . The temporary conversion of the wavelength grid to a fixed interval results in coefficients $c_{i}$ independent of the wavelength range and step size of the input spectrum. The wavelength solution is not changed if $c_{1}=1$ and $c_{i}=0$ for all other $i$. It is possible to set an individual start value for the constant term $c_{0}$ via the parameter CHEBY_CONST (see Table 1). In this way, significant possible shifts between the wavelength grids of the science and the sky spectrum can be considered.

The coefficients $c_{i}$ are determined by an iterative procedure. This process is initialised with two subsequent estimates (for a better $\sigma$-clipping) and a fit of the line flux correction factors (see Sect. 2.5). During this first iteration the wavelength grid remains untouched. In the next step, the coefficients $c_{0}$ and $c_{1}$ are fitted using MPFIT. Now, a new estimate is calculated and the line flux correction factors are fitted again. Then, the next iteration starts by fitting the wavelength grid, now applying a Chebyshev polynomial of degree 2. After that, the line scaling factors are adapted again to incorporate the change of the wavelength grid. Each iteration increases $n_{\mathrm{w}}$ by 1 and uses the results of the previous iteration as input. The search for the best polynomial degree is controlled by the three input parameters CHEBY_MIN, CHEBY_MAX, and WTOL (see Table 1). The iteration process is stopped once the highest polynomial degree given by CHEBY_MAX is reached. For a value of -1 , no wavelength grid correction is performed. The parameter CHEBY_MIN indicates the lowest degree, that is to say, the smallest number of iterations. For $n_{\mathrm{w}}$ not lower than CHEBY_MIN, the code checks whether the resulting $\chi^{2}$ shows a relative $\chi^{2}$ improvement of at least WTOL (default: $1 \times 10^{-3}$ ) compared with the best $\chi^{2}$, so far. If this is not the case, the procedure stops and the results for the polynomial with the lowest $\chi^{2}$ are taken. An exception is when CHEBY_MIN > CHEBY_MAX is chosen. In this case, the code runs until CHEBY_MAX is reached and the corresponding results for this degree are taken, regardless of the results for the lower polynomial degrees. The default values for CHEBY_MAX and CHEBY_MIN are 7 and 3.

Independent of the use of a Chebyshev polynomial, the modified sky spectrum has to be rebinned to the wavelength grid of the science spectrum. For this task, the code offers two options that can be selected by the parameter REBINTYPE (see Table 1). The first method sums the fractional fluxes of input pixels that contribute to the wavelength range of the output pixel. The second approach is based on the convolution of the input spectrum with a pixel-dependent asymmetric damped sinc kernel

$f(k)=\mathrm{e}^{-((k-s) / \delta)^{2}} \frac{\sin (\pi(k-s))}{\pi(k-s)}$,

with $k$ being an integer variable ranging from $-k_{\max }$ to $k_{\max }$. The damping constant $\delta$ and the kernel radius $k_{\max }$ are fixed and have the values 3.25 and 5. The parameter $s$ is the subpixel shift of the sky spectrum relative to the science spectrum. It is a function of the pixel position and ranges from -0.5 to 0.5 . For shifts higher than half a pixel, complete pixels are treated by a simple renumbering of the input pixels in the output spectrum. No convolution is performed for this integer part of the pixel shift. The approach is similar to that used in the IDL routine sshift2d.pro of the Lowell Buie Library ${ }^{8}$. However, the

\footnotetext{
8 http://www . astro.washington. edu/docs/idl/htmlhelp/ slibrary30.html
} 
original programme is only for a constant shift of the entire spectrum. A wavelength-dependent shift is also possible, as long as the shift changes slowly with the spectrum pixels and the pixel size is nearly constant for the whole input and output wavelength grids. These requirements are sufficiently met if inconsistencies of the wavelength grids are in the order of 1 pixel and if the functional dependence of the differences can be described by a low-order polynomial. The relatively complicated rebinning method described above is able to effectively suppress the broadening of spectral lines, which typically occurs if a spectrum is rebinned to a shifted grid of similar pixel size. The linebroadening suppression is achieved by alternating positive and negative contributions to the kernel as incorporated in the sinc shift method. Therefore, the sinc shift method produces the best results if significant subpixel shifts close to half a pixel are frequent. However, for a very good agreement of the wavelength grids with subpixel shifts close to zero, it might be better to use the simple rebinning method. In such a case, the relatively broad sinc kernel influences the spectrum more than simple regridding.

\section{Test data set}

To illustrate the performance of skycorr, we used data taken with the VLT echelle spectrograph X-shooter (Vernet et al. 2011). This instrument is well suited for testing sky subtraction, since its three arms UVB, VIS, and NIR cover the entire wavelength range affected by significant airglow emission. In fact, wavelengths from 0.3 to $2.5 \mu \mathrm{m}$ can be observed simultaneously with medium resolution between 3300 and 18200 depending on the arm and the selected slit widths. Since X-shooter is a slit spectrograph $^{9}$, the performance of skycorr can be compared with the classical sky-subtraction method based on the interpolation of 2D data.

We also investigated the skycorr performance for data taken with other instruments. The skycorr user manual that is provided along with the code also shows several VLT FORS and SINFONI examples.

\subsection{Sample selection and data reduction}

To analyse the sky-subtraction quality as a function of time, we searched for a time series of a single target in the ESO archive. We decided to use the observations of the ESO programme 288.D-5015, which was carried out on 25 Dec. 2011 between 0:58 and 3:25 UT and comprises 12 UVB, 24 VIS, and 80 NIR-arm spectra with exposure times of 606, 294, and $100 \mathrm{~s}$, respectively (see Set 1 in Table 4). The observed object was the ultracool white dwarf SDSS 0138-0016 (see Parsons et al. 2012). The spectrum is characterised by a maximum emission at about $1 \mu \mathrm{m}$, absorption bands in the near-IR, and also emission lines in the optical. As reference object spectrum to be corrected by skycorr, we defined the first observation in each arm. For all other observations, only the sky emission was used.

The X-shooter data were reduced using the ESO public pipeline release V2.0.0 executed with the Reflex workflow V2.3 (see Modigliani et al. 2010). To obtain the sky-subtraction results for the 2D interpolation method, we directly used the output pipeline 1D spectra. For the correction with skycorr, we reduced the data without sky subtraction and extracted the input spectra for skycorr from the resulting 2D spectra. For the object spectrum, we applied a wavelength-independent trace based

\footnotetext{
9 The IFU of X-shooter is an image slicer that converts a $4^{\prime \prime} \times 1.8^{\prime \prime}$ field into a $12^{\prime \prime} \times 0.6^{\prime \prime}$ pseudo-slit.
}

Table 4. Properties of the X-shooter test data set.

\begin{tabular}{lccc}
\hline \hline Par. & Set 1 & Set 2 & Set 3 \\
\hline ESO ID & $288 . \mathrm{D}-5015$ & 088.A-0725 & 086.A-0974 \\
$N_{\text {exp }}{ }^{a}$ & $12,24,80$ & $4,4,4$ & $7,7,7$ \\
Dates [UT] & $2011 / 12 / 25$ & $2011 / 12 / 19$ & $2010 / 12 / 29-31$ \\
Times $^{b}[\mathrm{UT}]$ & $00: 58-03: 25$ & $03: 12-04: 02$ & $05: 56-07: 31$ \\
Slit width $^{a}\left[{ }^{\prime \prime}\right]$ & $1.0,0.9,0.9$ & $1.0,0.9,0.9$ & $1.0,0.9,0.9$ \\
$T_{\exp }{ }^{a}[\mathrm{~s}]$ & $606,294,100$ & $705,609,246$ & $900,900,900$ \\
\hline
\end{tabular}

Notes. ${ }^{(a)}$ The number of exposures $N_{\text {exp }}$, the slit width, and the exposure time $T_{\exp }$ are given for the three X-shooter arms UVB, VIS, and NIR separated by commas. ${ }^{(b)}$ The time range was derived from the start of the first exposure and the end of the last exposure of all three arms. For Set 3, it was neglected that the observations had been performed on three different nights.

on the median object flux in spatial direction. Pixels masked by the pipeline were excluded from the extraction, and the summed object flux was scaled to correct for missing pixels using the object profile. This approach is similar to the method used by the pipeline. However, by doing it ourselves, we know the exact sky area that contributes to each wavelength. This information is important for extracting the sky from the 2D spectra. Here, we computed the median along the spatial direction and scaled the resulting spectrum to have the same sky area as the reference object spectrum. The data set was not flux calibrated, since the instrument response and the atmospheric extinction (the latter at least for the VIS and NIR arm) only slowly vary with wavelength and time compared with the defined airglow variability groups (see Sect. 2.4).

To test skycorr for sky data not taken in the same night as the reference object spectrum, we extended our sample by two additional data sets (see Sets 2 and 3 in Table 4). The observations had the same slit widths and long exposure times to avoid that the extracted median sky would be contaminated by a bright object. Set 2 was taken six nights earlier than the reference observations and comprises four spectra in each arm. Set 3 was taken about one year earlier in three subsequent nights and consists of seven exposures in each arm. Since the exposure times deviated from those of the reference data, the resulting 1D sky spectra were corrected for by the exposure time ratio.

\subsection{Sample properties}

Figure 10 provides an overview of the observing conditions for the investigated spectra. The data for each of the three subsamples (see Table 4) are displayed in separate panels as a function of the time interval between the mid-exposures of the sky and object observations.

The first row of panels (a) shows the change in airmass. The observation of a single target over $2.5 \mathrm{~h}$ in the main run results in a significant change in the airmass. This affects the airglow intensity because of the nearly proportional increase of the projected emission layer width (van Rhijn 1921; Noll et al. 2012).

The summed intensity of two P-branch lines of the $\mathrm{OH}(3-1)$ band is shown in the next row of panels. Apart from the airmassrelated van Rhijn effect, the intensity varies on different time scales because of changes of the chemical composition, temperature, and dynamics in the $\mathrm{OH}$ emission layer (see Khomich et al. 2008). For the entire data set, a highest ratio of 3 in the intensity can be observed, which illustrates the challenge in correcting for the sky in a science spectrum with data taken at a different 


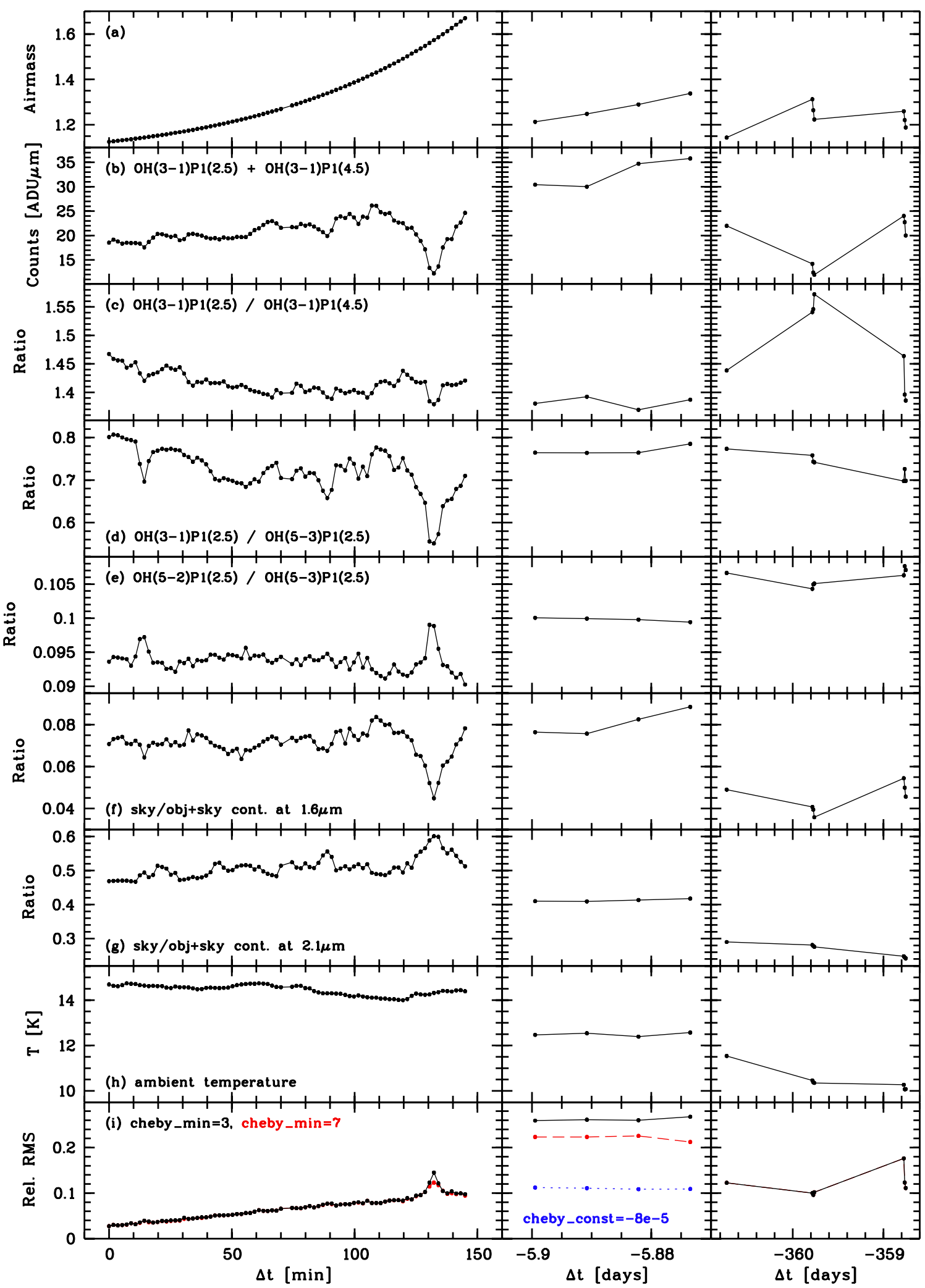

Fig. 10. Properties of the X-shooter NIR-arm test data and quality of the sky subtraction by skycorr as a function of time. The time difference $\Delta t$ is given relative to the object spectrum to be corrected for sky emission (2011/12/25 00:59:05 UT). The left, middle, and right panels characterise the sky data of the same night, about six nights before, and about one year before the object reference exposure, respectively. From top to bottom, the panels display a) the airmass, b) the summed intensity of two airglow lines of the $\mathrm{OH}(3-1)$ band, c) the ratio of the same lines tracing relative $B$-group variability, d) the ratio of the same rotational line in two different $\mathrm{OH}$ bands tracing the relative $A$-group variability, e) the ratio of two $\mathrm{OH}$ lines, which can only vary by effects unrelated to airglow, $\mathbf{f}$ ) the sky continuum at $1.6 \mu \mathrm{m}$ relative to the corresponding measurement in the uncorrected object spectrum, $\mathbf{g}$ ) a similar ratio for $2.1 \mu \mathrm{m}, \mathbf{h}$ ) the ambient temperature in $\mathrm{K}$, and i) the rms of the sky-subtraction residuals for line pixels relative to the mean line peak flux. The latter is shown for two different minimum degrees of the Chebyshev polynomial for the improvement of the wavelength solution by skycorr. In the middle panel of i), the effect of an initial shift of the wavelength scale by one pixel is also plotted (dotted line). 
time. The variations are not only caused by airglow variability. Changes of the instrument performance and calibration data can also have an effect. Moreover, variations in the atmospheric transparency can be important for non-photometric observations. This explains the dip in the intensity about $135 \mathrm{~min}$ after the start of the main run, when a small cloud probably covered the sky in the line of sight ${ }^{10}$. Note that the decrease of the airglow intensity was moderate compared with the almost complete extinction of the object light. The difference is caused by the fact that the observed star is a point source, whereas airglow emission covers the entire sky. This allows a partial compensation of extinction losses by scattering of light from other sky positions into the line of sight (see Chamberlain 1961; Noll et al. 2012). Running skycorr with a sky spectrum taken under such unfavourable conditions is a good test of the applicability of the method in challenging situations.

Panels (c) to (e) of Fig. 10 show the time dependence of different line ratios. They indicate a weaker variability than the intensity. However, variations in the order of $10 \%$ for $\mathrm{OH}$ can still be critical for a reasonable correction of strong airglow lines. For this reason, the line groups described in Sect. 2.4 were introduced. While (c) shows an example of the time-dependent deviations between different $B$ groups, (d) and (e) are examples of deviations in the intensity ratios of $A$ groups. Since the bands in (e) have the same upper vibrational level $v^{\prime}=5$, the ratio should be constant if the airglow variability alone is considered. Therefore, the significant changes (especially if different observing runs are compared) trace the influence of instrumental effects and atmospheric transparency.

Panels (f) and (g) show the ratio of the sky and object+sky continuum for the wavelengths 1.6 and $2.1 \mu \mathrm{m}$, respectively. Since the sky continuum cannot be adapted by skycorr (see Sect. 2), the variation of such ratios is critical for the quality of the continuum correction. In the $H$ band, the sky continuum is weak. Except for the cloud event and the observations that were taken about one year before the object spectrum, the variation of the sky relative to the object is in the order of $1 \%$ only. Hence, continuum errors by the sky subtraction in the $H$ band should be small as long as the observed object is not distinctly fainter than the investigated white dwarf. In the $K$ band, the thermal emission by the telescope is a strong component. For our test data, the contribution is up to $60 \%$ at $2.1 \mu \mathrm{m}$, which means that the telescope emission is about as strong as the object emission. The flux ratio in $(\mathrm{g})$ is well correlated with the ambient temperature in $(\mathrm{h})$, which determines the mirror temperature. The temperature deviated by up to $4.6 \mathrm{~K}$ from the value of $14.7 \mathrm{~K}$ for the reference exposure. Because of the strong variability, a good thermal continuum subtraction appears to be difficult to achieve in this wavelength regime if an object is not significantly brighter than the thermal emission, and/or the temperature differences between the object and sky exposures are not distinctly lower than $1 \mathrm{~K}$. At least in the case of $\mathrm{X}$-shooter, the strong variations in the $K$ band might also partly be related to instabilities of the flat-field lamps (Vernet, priv. comm.). Hence, the continuum subtraction for Set 2 and 3 could be improved by using the same flat-field as for Set 1. For flux-calibrated spectra, which we did not analyse, the use of the same flat-field during the reduction of the science and standard star spectra is the default setting in the X-shooter pipeline Reflex workflow.

\footnotetext{
${ }_{10}$ This interpretation is consistent with data from ESO's Ambient Conditions Database.
}

\section{Results}

In the following, we evaluate the performance of skycorr on the data set discussed in Sect. 3. At first, the test approach is described (Sect. 4.1). Then, the performance of skycorr is compared with the 2D sky interpolation method applied by the $\mathrm{X}$-shooter pipeline (Sect. 4.2). This test can only be carried out if there is no time difference between the science and the sky spectra. Next, the performance is analysed for different time intervals by using the entire test data set (Sect. 4.3). Finally, we compare the results of skycorr with those obtained without line scaling (Sect. 4.4) and using the method of Davies (2007) for our test data (Sect. 4.5).

\subsection{Test approach}

For the test runs, we used a fixed set-up of the parameters listed in Table 1. Since skycorr was optimised to minimise user interaction, most parameter values agree with the listed default values. The parameter VAC_AIR was set to "air" because the $\mathrm{X}$-shooter pipeline provides wavelengths in air. Since the $\mathrm{X}$-shooter spectra have a sufficiently high resolution, some parameters can be modified to speed up the code without deteriorating the results. Setting FLUXLIM $=0.005$ switches off the iterative search of continuum windows between the lines (see Sect. 2.1) without changing the result. We also modified the FWHM and $\chi^{2}$ convergence criteria LTOL $\left(1 \times 10^{-1}\right)$ and FTOL $\left(1 \times 10^{-2}\right)$, which did not negatively affect the sky-subtraction quality. For X-shooter NIR-arm spectra with about 25000 pixels, the run times were measured to be between 12 and $25 \mathrm{~s}^{11}$, mainly depending on the number of calculated polynomials of different degrees (see Sect. 2.6). Without changing the parameters discussed above, the run times would have been between 12 and $36 \mathrm{~s}$. Since X-shooter spectra cover a wide wavelength range that is characterised by a roughly linear increase of the width of the line profile in pixels, we set VARFWHM to 1 (see Sect. 2.2). Finally, we reduced SIGLIM (Sect. 2.5) from the default value 15 to 5 , since the test spectra show many outliers due to bad pixels and not all of them were masked by the pipeline.

Skycorr produces an output ASCII file that includes the best-fit parameters and several other quantities that can be used to evaluate the sky-subtraction quality. In particular, the file provides the rms of the error-weighted sky-subtraction residuals (no continuum) for line pixels relative to the error-weighted mean line peak flux. The weights depend on the statistical noise and possible systematic errors by bad pixels and similar defects. The selection of line pixels originates from the line-continuum separation discussed in Sect. 2.1. The relative rms, as defined above, has turned out to be a good indicator of the sky line subtraction quality and is used in the following. Good values are in the order of a few per cent.

\subsection{Comparison with the $2 D$ sky interpolation method}

As a first test of the quality of the sky subtraction by skycorr, we compare the results for $\Delta t=0$, that is to say, the science and sky spectrum taken from the same 2D spectrum, with those of the X-shooter pipeline. Figure 11 shows these resulting spectra in the three X-shooter arms. Both methods produce convincing spectra of the target star (cf. Parsons et al. 2012). In the UVB arm $(0.30-0.59 \mu \mathrm{m})$, stellar emission lines can be seen. The VIS

\footnotetext{
${ }^{11}$ Run on a Core2Quad Q9550@2.83GHz,8GB RAM, Fedora 19 (64 bit).
} 


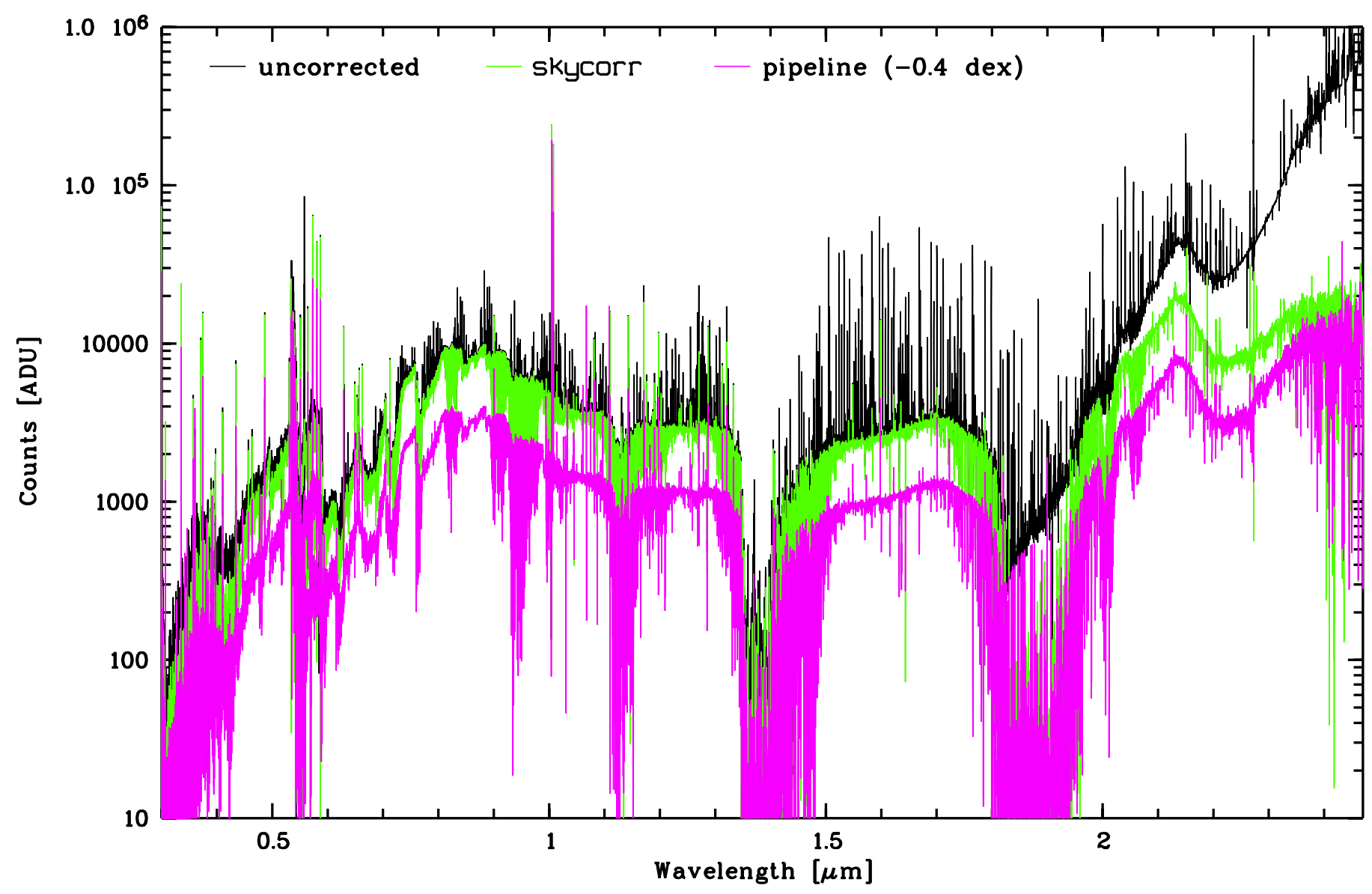

Fig. 11. Sky-subtraction results for the three X-shooter arms for the sky spectrum derived from the same 2D spectrum as the object spectrum $(\Delta t=0)$. The input object spectrum (black), the skycorr-corrected spectrum (green), and the corresponding X-shooter pipeline product based on sky interpolation in the 2D spectrum (magenta) are shown. For a better comparison, the latter is shifted by 0.4 dex to lower values. The spectra are not flux calibrated. This explains the apparent flux jump between the UVB and VIS arm at about $0.59 \mu \mathrm{m}$ and the bump at about $2.14 \mu \mathrm{m}$ (cf. Parsons et al. 2012). The strong uncorrected lines in the near-IR (mainly between 1.0 and $1.35 \mu \mathrm{m}$ ) were mostly caused by instrumental defects.

$\operatorname{arm}(0.53-1.02 \mu \mathrm{m})$ mainly reveals stellar absorption bands, and the NIR arm $(0.99-2.48 \mu \mathrm{m})$ shows strong atmospheric absorption of the stellar continuum (essentially by water vapour bands). Note that the spectra are not flux calibrated (see Sect. 3.1). The UVB and VIS-arm spectra are only affected by relatively weak sky features compared with the object continuum (cf. Sect. 2.4). Therefore, a good sky correction is not particularly difficult for the given example. The situation is much more challenging in the $\mathrm{X}$-shooter NIR-arm range, where the sky emission tends to be significantly brighter, reaching up to 1 to 2 orders of magnitude higher intensities than the stellar continuum. For this reason, we focus the tests in Sect. 4 on the NIR arm.

Despite the strong airglow lines, skycorr and the 2D interpolation method produce sky-corrected spectra with relatively smooth object continua. The strongest residuals can be identified as instrumental defects (bad pixels). The most obvious difference between the results of both methods is the deviating continua at the red margin of the NIR arm. This discrepancy can be explained by the very strong thermal background, intensity gradients along the spatial direction in the 2D spectra, and the differences in the extraction of the 1D spectra (see Sect. 3.1). Thus, the deviations are not related to the sky-subtraction quality. For a more quantitative comparison of this quality, we calculated the relative rms defined in Sect. 4.1. Interestingly, the skycorr output NIR-arm spectrum has a significantly smaller rms (0.027) than the corresponding pipeline spectrum (0.040). This result is robust. Modifying the considered wavelength range or the exclusion algorithm for unreliable rms outliers (bad pixels) did not change it significantly. Even though this is just one example and the approach for the extraction of the 1D spectra might influence the rms, skycorr appears to be able to subtract sky emission lines at least as well as a method that benefits from the full 2D spectral information.

\subsection{Effect of time differences between science and sky spectra}

The main purpose of skycorr is the subtraction of the sky emission in science spectra by means of sky data taken at a different time and sky position. Using the data set described in Sect. 3.1, the quality of this correction can be investigated as a function of time. In Sect. 3.2, we have discussed the sky variability during the selected observing runs on the basis of the data plotted in Fig. 10. The last row of this figure shows the relative rms (see Sect. 4.1) for the NIR-arm spectra of the test data set. For the skycorr parameter set-up listed in Table 1 (black symbols and lines), the left-hand panel indicates a gradual increase of the rms from 0.027 at the beginning, over 0.067 at half of the time, to 0.098 at the end of the run. A special situation occurred at $\Delta t \approx 132$ min with a maximum rms of 0.145 . As discussed in Sect. 3.2, a cloud probably covered the sky in the target direction. This caused a significant decrease of the sky-subtraction 


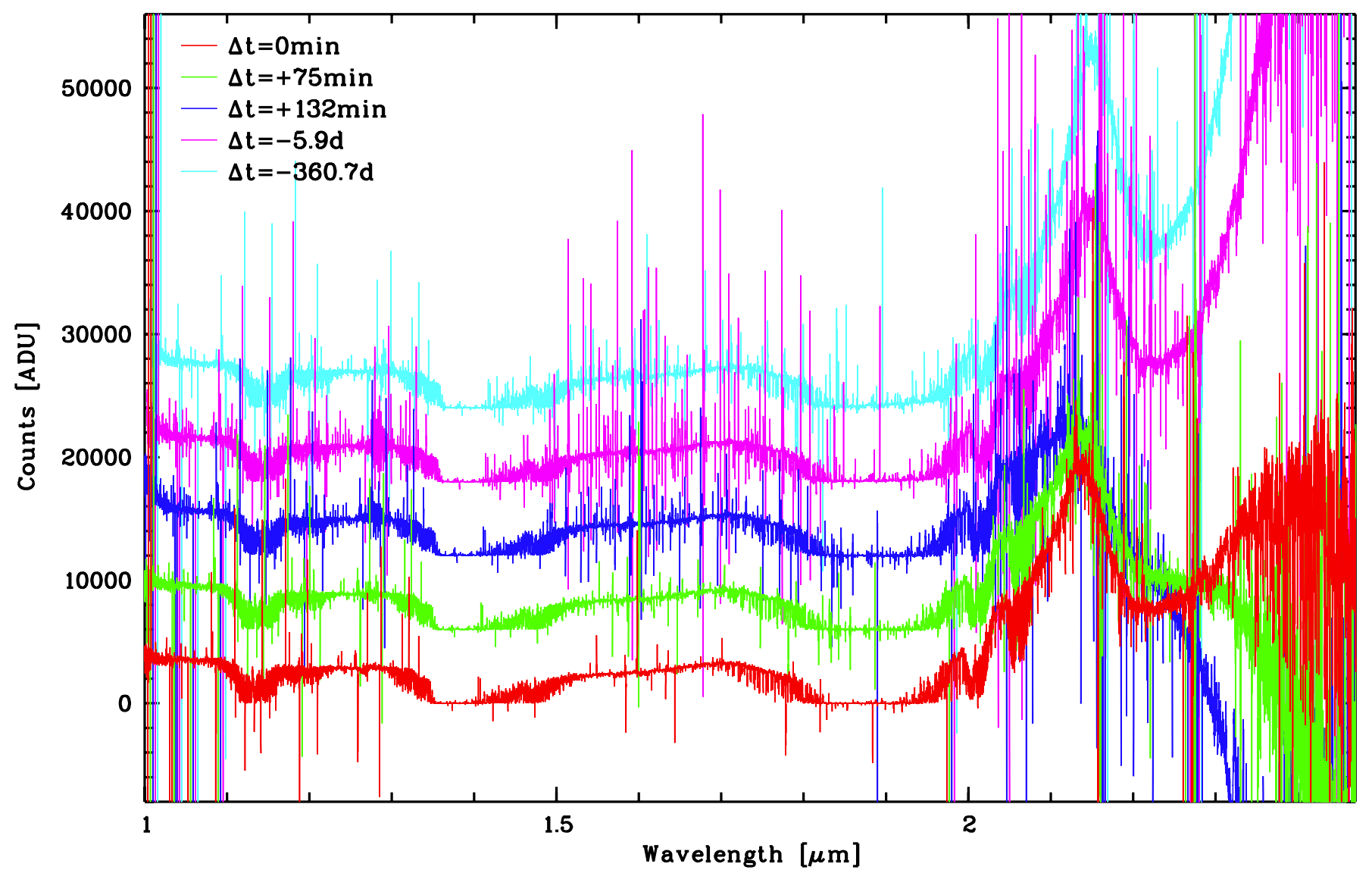

Fig. 12. Skycorr output spectra for different X-shooter NIR-arm data sets consisting of the same input object spectrum, but different sky spectra. The colour codes for the various time differences are given by the figure legend. For a better comparison, adjacent spectra are shifted by 6000 ADU and $3 \mathrm{~nm}$.

quality. However, these results are still better than the sky correction performed with sky data taken six nights before. Here, the relative rms was about 0.26 . The most noticeable difference in the atmospheric conditions with the reference object exposure was the $\mathrm{OH}$ emission intensity (middle panel in row (b)), which was almost twice as high. This probably contributes to the poorer performance of skycorr. However, a long time difference does not inevitably mean a poor sky-correction quality, as Set 3 in the right-hand panel demonstrates. Although the time interval was about one year, the rms values are between 0.10 and 0.18 , which is significantly better than for $\Delta t=-6 \mathrm{~d}$ and even comparable with the end of the main run. The $\mathrm{OH}$ intensity for Set 3 was closer to the reference value than for Set 2, but the discrepancies are still quite large. Moreover, rows (d) to (f) of Fig. 10 suggest significant differences in the instrumental properties and calibration (see Sect. 3.2).

To better understand the skycorr performance differences, Fig. 12 shows the sky-subtracted NIR-arm spectra for five different cases: $\Delta t=0 \mathrm{~min}$ (the reference exposure), $+75 \mathrm{~min}$ (half of the time of the main run), +132 min (cloud), $-5.9 \mathrm{~d}$ (first exposure of Set 2), and $-360.7 \mathrm{~d}$ (first exposure of Set 3 ). In addition, Fig. 13 displays the input sky lines and the corresponding sky-subtraction residuals for the single strong airglow line $\mathrm{OH}(3-1) \mathrm{P} 1(2.5)$ (see Sect. 2.4). Figure 14 shows a similar plot for the only partly resolved $\mathrm{O}_{2}(\mathrm{a}-\mathrm{X})(0-0)$ band, which is also blended with $\mathrm{OH}(8-5)$ lines. The figures confirm that the strongest sky line residuals are found for the cloud event and Set 2. The sky-corrected spectrum for the Set 3 example is an intermediate case. Further implications are discussed in the following paragraphs.
Concerning the continuum correction, the cloud event changed the thermal background in a way that the sky-corrected object continuum is completely wrong in the red part of the $K$ band (Fig. 12). Significant changes in the background can already be observed for $\Delta t=75 \mathrm{~min}$. Since the sky continuum cannot be adapted to changing observing conditions, the continuum correction especially in the red part of the $K$ band has to be taken with care if the object is distinctly fainter than the background (see Fig. 11).

As already indicated by Fig. 10, the intensities of the $\mathrm{OH}$ lines deviated the most from the reference exposure for the cloud event and Set 2. Figure 13 also reveals that these cases show a significant shift of the line centres. In particular, the Set 2 spectrum is shifted by about one pixel. Since the physical changes of airglow line positions are orders of magnitude too small to be visible in the X-shooter data, the shifts are probably caused by instrumental effects related for example to the position of the orders on the chip, variations in the wavelength calibration frames, or uncertainties in the determination of the wavelength solution by the pipeline. As the strong asymmetric residuals for the critical cases suggest, the wavelength shifts appear at least as important for the sky-subtraction quality as the intensity of the lines. Skycorr corrects the input wavelength solution by fitting Chebyshev polynomials, where the degree is increased in an iterative procedure (see Sect. 2.6). The default algorithm is a search that at least checks degree 3 and at most degree 7 (see Table 1). Interestingly, the critical cases were only checked up to the minimum degree CHEBY_MIN $=3$. Therefore, we ran the code for CHEBY_MIN $=7$, which significantly increased the code run time, but also improved the rms for the 


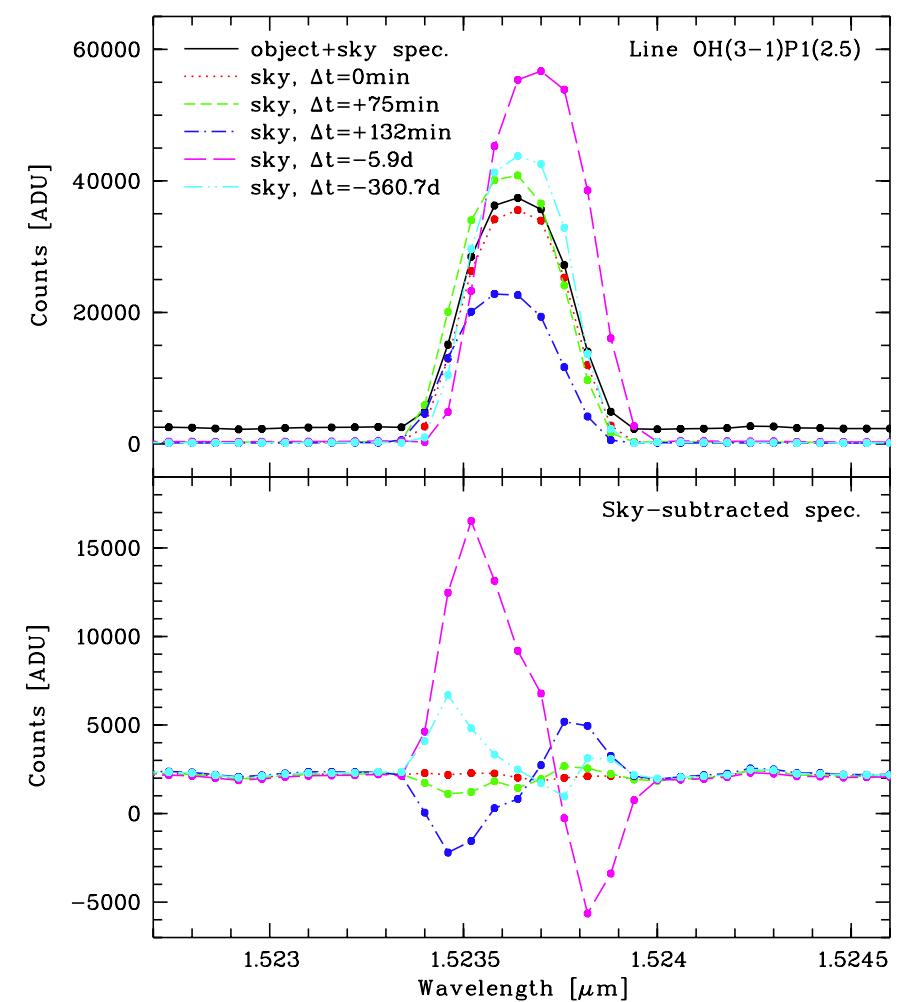

Fig. 13. Subtraction of the strong airglow line $\mathrm{OH}(3-1) \mathrm{P} 1(2.5)$ for several time intervals between the object and the sky spectrum. The upper panel shows the line in the input object (black) and sky spectra (see legend for colour and line type). The lower panel displays the corresponding sky-subtraction residuals. To enhance their visibility, the ordinate was significantly zoomed.

cloud event and Set 2, as Fig. 10 indicates (red symbols and lines in last row). Surprisingly, the best fit for the critical cases was achieved without changing the input wavelength solution. Hence, the iterative procedure appeared to mainly improve the fitting of the line intensities. The parameter CHEBY_CONST (see Table 1) allows a constant initial shift of the wavelength grid. By setting it to $-8 \times 10^{-5}$, which corresponds to about one pixel for X-shooter NIR-arm spectra, we can compensate for the shift found for the Set 2 example. The resulting rms is also shown in Fig. 10. For the selected Set 2 spectrum, it decreased from 0.26 to 0.11 . This striking improvement suggests that the fitting procedure of the standard run did not find the global $\chi^{2}$ minimum because of strong differences in the airglow intensities and wavelength grids of the input science and sky spectra. In such critical cases, the skycorr parameter set-up can be optimised.

For blended $\mathrm{O}_{2}$ lines, Fig. 14 indicates that the most significant residuals are again found in the cloud case and Set 2. Nevertheless, the situation is different since the $\mathrm{O}_{2}$ lines showed a completely different time dependence as the $\mathrm{OH}$ lines. This can be seen at wavelengths beyond $1.275 \mu \mathrm{m}$, where both kinds of lines are present. At $\Delta t=0$, the $\mathrm{O}_{2}$ band was distinctly brighter than for all other examples plotted. In particular, the observations of Sets 2 and 3 showed intensities that were lower by a factor of 5 to 6 . In view of the required strong intensity corrections and the blending of lines with different variability, the quality of the recovered stellar continuum is remarkably good. Only the spectrum related to the cloud shows significant continuum offsets at the wavelengths where line blending is most critical. The target spectrum retrieval can be more difficult than in the present case if the observed object has a complex spectrum in the

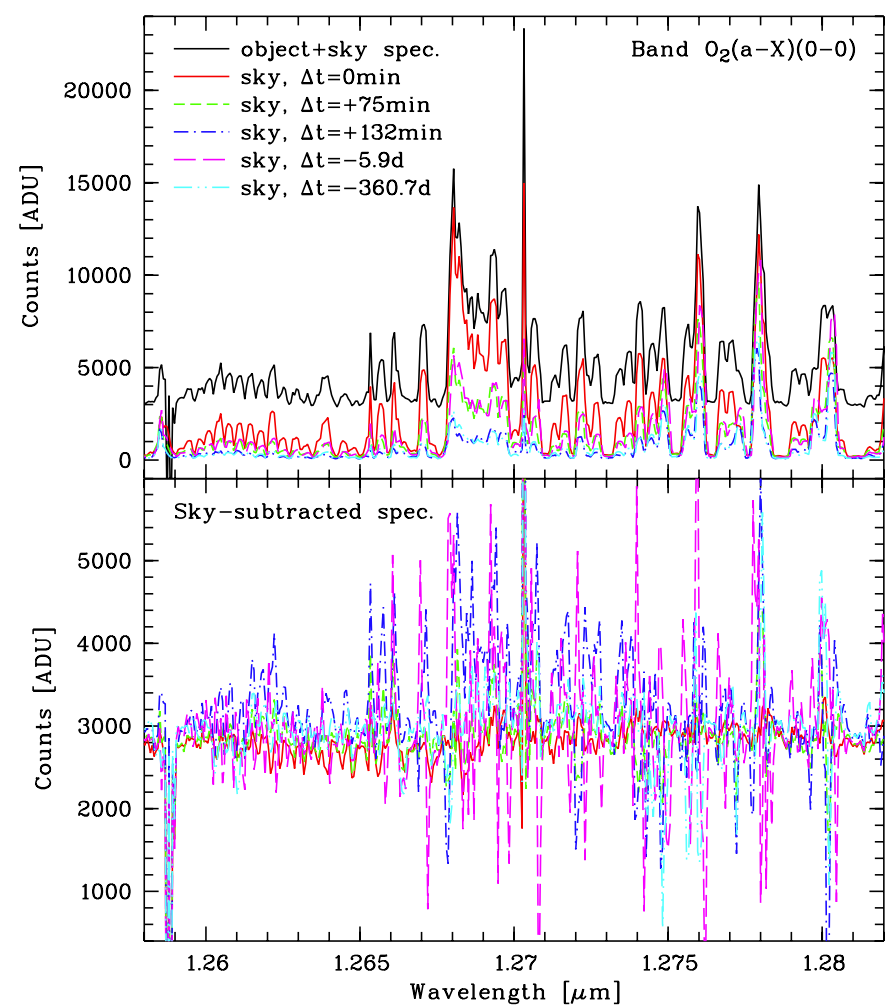

Fig. 14. Subtraction of the strong and only partly resolved airglow band $\mathrm{O}_{2}(\mathrm{a}-\mathrm{X})(0-0)$ (cf. Fig. 8) for several time intervals between the object and the sky spectrum. The upper panel shows the band in the input object (black) and sky spectra (see legend for colour and line type). The lower panel displays the corresponding sky-subtraction residuals. Note that the ordinates are different in both panels. The two strong uncorrected lines in the centre and the left margin of the figure were caused by instrumental defects. Longwards of $1.275 \mu \mathrm{m}$, the $\mathrm{O}_{2}$ lines are blended with $\mathrm{OH}(8-5)$ lines.

range of the $\mathrm{O}_{2}$ band. Then, the separation of blended sky lines and continuum could be systematically wrong, which would be a problem if the airglow lines need to be scaled by factors far from 1 . The sky line subtraction at about $1.27 \mu \mathrm{m}$ is certainly the most difficult for X-shooter. For lower resolution spectra, additional wavelength ranges with blended sky lines could have similar problems.

The NIR-arm spectrum of SDSS 0138-0016 (see Fig. 11) does not allow the test of how object emission lines would appear after the subtraction of strong sky lines at similar wavelengths. For this reason, we carried out a challenging test by multiplying the strong $\mathrm{OH}$ line in Fig. 13 by 1.5 in the object spectrum and running skycorr with the full data set for this modified input spectrum. This modification simulates an object emission line with the position, shape, and $50 \%$ of the intensity of the $\mathrm{OH}$ line. Figure 15 shows the sky-subtraction results for the five selected cases compared with the expected object spectrum. Apart from the $\Delta t=0$ and $75 \mathrm{~min}$ cases, which indicate an almost perfect line reconstruction, the Set 3 spectrum taken almost one year before the reference spectrum also shows a convincing line profile. This result suggests that the conservation of object lines by means of the line grouping of skycorr (see Sect. 2.4) works in general. Unsatisfying line profiles are only found for the two difficult cases. The quality of the sky subtraction depends on the differences between the sky in the input science and sky spectra as well as on the ratio of the object and sky line intensities. 
S. Noll et al.: Skycorr: A general tool for spectroscopic sky subtraction

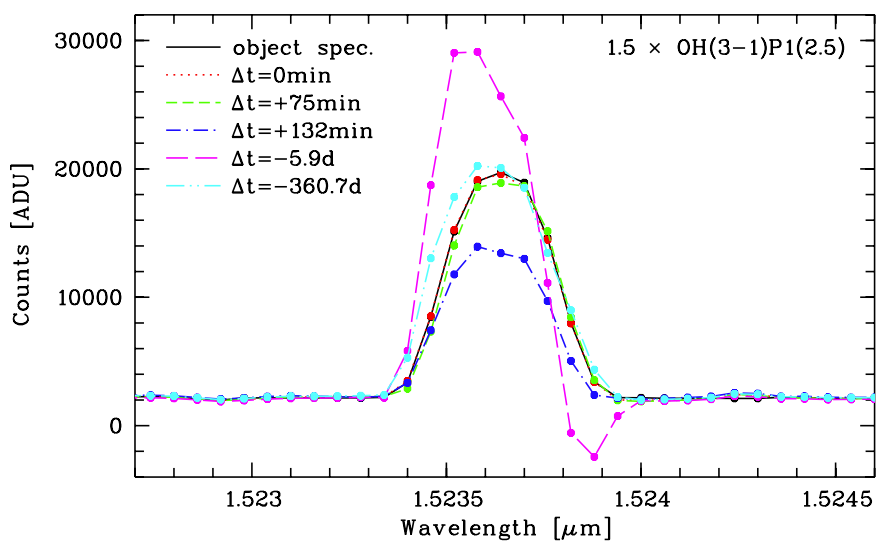

Fig. 15. Subtraction of the strong $\mathrm{OH}(3-1) \mathrm{P} 1(2.5)$ line from an object spectrum with an artificial emission line (black) at the same position and with the same line shape but only $50 \%$ of the strength of the sky line. The sky-correction results are shown for several time intervals between the object and the sky spectrum (see legend for colour and line type).

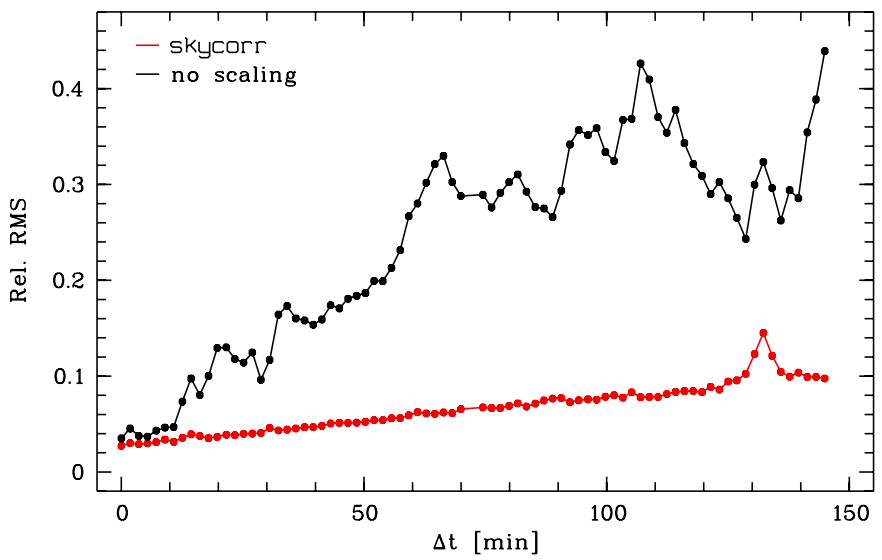

Fig. 16. Comparison of the rms of the sky-subtraction residuals relative to the mean line peak flux (see Sect. 4.1) by skycorr (red) and without line scaling (black) for the data set of the X-shooter NIR-arm sky spectra taken in the same night as the corrected object spectrum. The results are shown as a function of the time difference between the object and the sky spectrum.

\subsection{Comparison with sky subtraction without scaling}

Figure 16 shows the resulting rms of Set 1 for skycorr (cf. Fig. 10) and the sky spectrum just subtracted from the science spectrum without scaling. In the first $10 \mathrm{~min}$, the rms was only slightly higher than for skycorr (ratios between 1.2 and 1.5). This indicates that for time intervals of a few minutes, a significant correlation of the sky line intensities can be assumed. Afterwards, the rms ratio was quickly increasing, reaching ratios up to 5.5. For Sets 2 and 3, the rms ratios ranged from 3.3 to 4.1 and from 2.4 to 5.1. These factors demonstrate that skycorr performs significantly better than a simple on-off technique without sky scaling. They also show that the latter method is only reliable for very short time differences.

\subsection{Comparison with the Davies method}

As far as we know, the method of Davies (2007) is the only existing approach with a similar philosophy and purpose as skycorr. For this reason, it is prudent to compare the performance of this method and skycorr. To this end, we attempted to run Davies' IDL code for the X-shooter NIR-arm test data set defined in

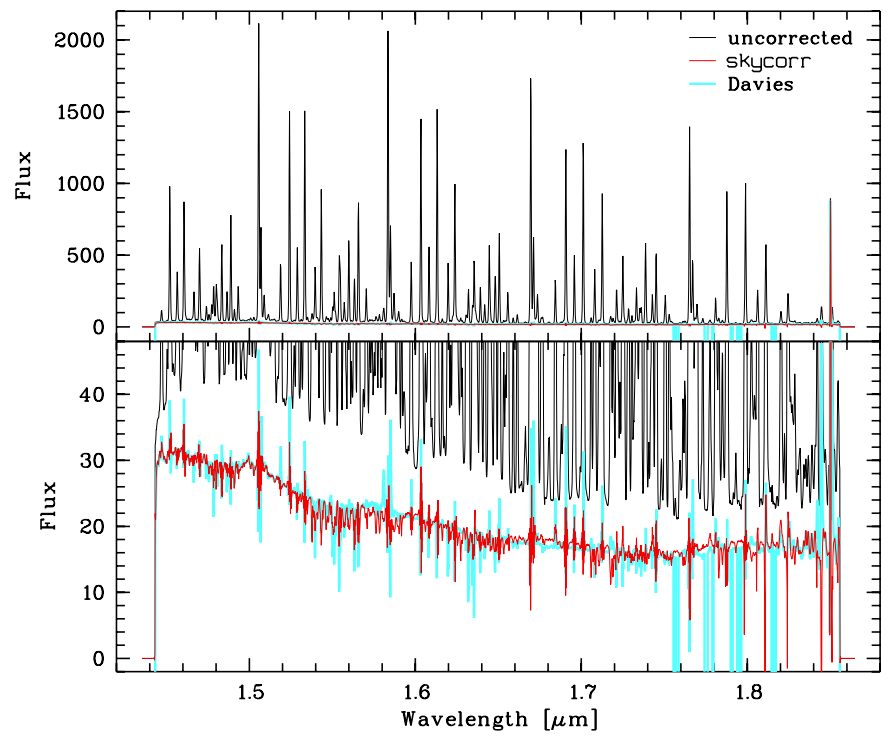

Fig. 17. Comparison of skycorr (red) and Davies' code results (cyan) for an example SINFONI $H$-band spectrum of the ESO observing programme 083.B-0456. While the upper panel shows the full input science spectrum (black), the lower panel focuses on the sky-subtraction results. Highly negative fluxes (only Davies' code) mark pixels with undefined fluxes. The time difference between the object and sky exposures was $39 \mathrm{~min}$. Note that the faint object spectrum was derived from the sky cube. The originally targeted object could not be detected.

Sect. 3.1. Since the version 1.1 implemented in the currently released ESO SINFONI pipeline (Modgiliani et al. 2007) is already fairly old, we improved the results by using the most recent version 2.0. Since SINFONI is an IFU spectrograph (Eisenhauer et al. 2003), 3D data cubes with the spectra of a spatial 2D grid are required as input. However, Davies' code also performs the sky line scaling on 1D spectra that are derived from the filtered and integrated cubes for object and sky observations. By replacing the internal 1D arrays for object and sky by the X-shooter input data and writing the sky-subtracted object array into a file (neglecting the subsequent application of the best solution to the object cube), we were able to run Davies' code and to compare it with skycorr. The runs were performed with default parameters plus the thermal background subtraction and the wavelength scale correction (via cross correlation) switched on.

The test indicates that skycorr outperforms Davies' algorithm for X-shooter data. The SINFONI-optimised Davies' code appears to be unstable for the investigated data because of an erratically varying quality of the correction. Possible reasons are the much wider wavelength range than that of SINFONI, the higher resolution, the low $\mathrm{S} / \mathrm{N}$ ratio, the numerous bad pixels, the use of non-flux-calibrated spectra, and a complex continuum that cannot be fitted by a single greybody. Despite the higher complexity, skycorr was also faster than the 1D part of Davies' code, which might be related to the use of $\mathrm{C}$ instead of IDL.

For a fair comparison of skycorr and Davies' method, we also considered SINFONI spectra, for which the latter method was designed and works reliably. To illustrate the performance of both codes for SINFONI data, we studied an $H$-band (Fig. 17) and a $K$-band example (Fig. 18). Since we are interested in the results for significant time intervals, we did not use the sky cubes that belong to the object cubes. Instead, we took the sky cubes from other observations of the same observing programmes. In this way, time differences of 39 and $11 \mathrm{~min}$ for the $H$ and $K$ band could be achieved. The data were reduced with ESO's SINFONI 


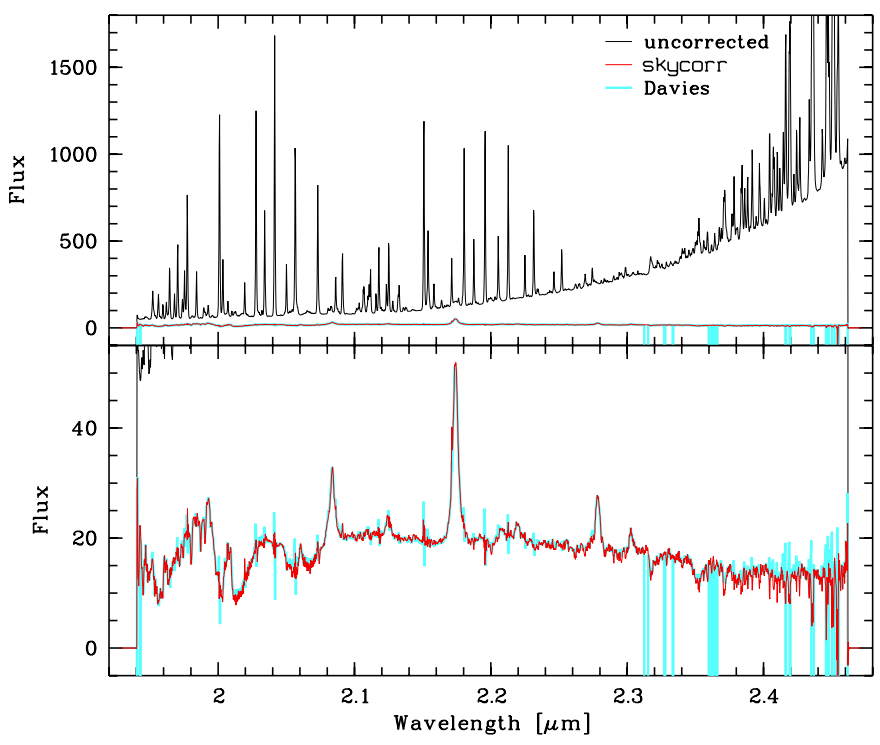

Fig. 18. Comparison of skycorr (red) and Davies' code results (cyan) for an example SINFONI $K$-band spectrum of the ESO observing programme 075.B-0648 showing the Seyfert 2 galaxy NGC 6240 (cf. Rosenberg et al. 2012). While the upper panel shows the full input science spectrum (black), the lower panel focuses on the sky-subtraction results. Highly negative fluxes (only Davies' code) mark pixels with undefined fluxes. The time difference between the object and sky exposures was $11 \mathrm{~min}$.

pipeline. However, we used version 2.0 of Davies' code for the sky subtraction, as already mentioned above. The code flags were the same as for the X-shooter data set. To run skycorr and the comparison in Figs. 17 and 18, we used the 1D spectra produced by Davies' code.

For both examples, the two codes show a good performance, even though the objects are up to two orders of magnitude fainter than the sky. Nevertheless, the skycorr sky line residuals are significantly weaker. For shorter time intervals, the quality of the sky subtraction tends to be more similar, as other tests indicated. The corrected object continua show only slight deviations. For longer time intervals, this might change, as both codes use different algorithms for the continuum correction (see Sects. 1 and 2.3).

In conclusion, the algorithms implemented in skycorr seem to result in a consistently better sky subtraction. Our code is more robust and flexible, which means that it can reliably be applied to data of different instruments and set-ups.

\section{Conclusions}

We have presented a new method to subtract the sky in 1D spectra by means of optimised plain-sky spectra taken at different times and sky positions. This method, which will be provided to the community as software package skycorr, has been inspired by the approach described by Davies (2007), but it is more sophisticated. Important features are an iterative separation of sky lines and continuum, detailed line grouping based on an airglow model, pixel-based scaling of line groups, and an adaptation of the wavelength solution of the input sky spectra by Chebyshev polynomials and asymmetric damped sinc kernels. The sky correction of an input science spectrum consists of the subtraction of the best-fit scaled airglow lines and the separated continuum of the reference sky spectrum.

We have tested the performance of the instrumentindependent code by means of X-shooter spectra. Fixing the object spectrum to be corrected, we have analysed the skysubtraction quality for different time intervals between the science and plain-sky spectra. This comparison revealed promising results that depend on the change of the airglow intensity, atmospheric transparency, and the instrument calibration. The latter includes changes of the instrument sensitivity and especially the wavelength calibration. Although the best corrections are possible for time intervals of only a few minutes, where the airglow intensities are still very similar, convincing sky corrections were even achieved for time differences of about one year. The skycorr results were also compared with the 2D sky interpolation method of the X-shooter pipeline, Davies' method, and the simple on-off method without sky fitting. In conclusion, skycorr performed at least as well as the 2D approach and convincingly better than Davies' SINFONI-optimised code. The latter result was confirmed for two SINFONI examples and is due to the more complex sky optimisation of skycorr, the more robust performance, and the high flexibility in terms of the properties of the input data. Compared with sky subtraction without fitting, the skycorr sky line residuals for the test data set without parameter optimisation were between 2.1 and 5.5 times lower if short time intervals of a few minutes were neglected.

These promising results suggest that skycorr can be a valuable tool for wavelength ranges where airglow emission lines dominate the sky background and instrument set-ups that either offer only $1 \mathrm{D}$ data or require additional observations if the 2D science exposure does not provide plain sky. Even for sky subtraction in multi-object spectra without time differences, it might be useful if instrument parameters, such as the slit width and the wavelength coverage, are the same for object and sky spectra.

Skycorr has been developed for Cerro Paranal. However, it is feasible to use it for spectra taken at other observing sites. The specific Cerro Paranal airglow variability model is mainly used for setting the initial weights for the different line groups for each pixel. Since this model only considers five classes, it could only have an influence if there was a significant contribution for instance of an $\mathrm{OH}$ and $\mathrm{O}_{2}$ line to a certain pixel. This is relatively rare. Moreover, pixels with a strong blending of line groups are usually excluded from the fitting procedure. Ratios of lines of the same molecule as provided by the model line list can also vary depending on the observing site. However, these changes are distinctly weaker than the overall intensity variations and hardly relevant for the weights. Finally, short-term intensity variations can cause significant deviations from the airglow model. Therefore, any intensity prediction is relatively rough, even for well-studied observing sites. In any case, the largest impact on the sky-subtraction quality is that of the reference sky spectra, which have to be taken individually for each instrumental set-up.

Acknowledgements. We thank R. Davies for providing his IDL code. We are also grateful to the anonymous referee for his/her detailed and valuable comments. This project made use of the ESO archive facility. X-shooter and SINFONI data of the programmes 075.B-0648, 083.B-0456, 086.A-0974, 088.A-0725, and 288.D-5015 were analysed. This study was carried out in the framework of the Austrian ESO In-Kind project funded by BM:wf under contracts BMWF10.490/0009-II/10/2009 and BMWF-10.490/0008-II/3/2011. This publication is also supported by the Austrian Science Fund (FWF). S. Noll receives funding from the FWF project P26130. W. Kausch is also funded by the project IS538003 (Hochschulraumstrukturmittel) provided by the Austrian Ministry for Research (bmwfw).

\section{References}

Bates, D. R., \& Nicolet, M. 1950, J. Geophys. Res., 55, 301

Chamberlain, J. W. 1961, Physics of the Aurora and Airglow (New York: Academic Press) 
Clough, S. A, Shephard, M. W., Mlawer. E. J., et al. 2005, J. Quant. Spectrosc. Radiat. Transf., 91, 233

Cosby, P. C., Sharpee, B. D., Slanger, T. G., Huestis, D. L., \& Hanuschik, R. W. 2006, J. Geophys. Res., 111, A12307

Davies, R. I. 2007, MNRAS, 375, 1099

Dekker, H., D’Odorico, S., Kaufer, A., Delabre, B., \& Kotzlowski, H. 2000, in Optical and IR telescope instrumentation and detectors, Proc. SPIE, 4008, 534

Eisenhauer, F., Abuter, R., Bickert, K., et al. 2003, in Instrument Design and Performance for Optical/Infrared Ground-based Telescopes, eds. I. Masanori, \& A. Moorwood, Proc. SPIE, 4841, 1548

Ellis, S. C., \& Bland-Hawthorn, J. 2008, MNRAS, 386, 47

Goldman, A., Schoenfeld, W. G., Goorvitch, D., et al. 1998, J. Quant. Spectrosc. Radiat. Transf., 59, 453

Hanuschik, R.W. 2003, A\&A, 407, 1157

Jones, A., Noll, S., Kausch, W., Szyszka, C., \& Kimeswenger, S. 2013, A\&A, 560, A91

Khomich, V. Y, Semenov, A. I., \& Shefov, N. N. 2008, Airglow as an Indicator of Upper Atmospheric Structure and Dynamics (Berlin: Springer)

Meinel, A. B. 1950, ApJ, 111, 555
Mies, F. H. 1974, J. Molec. Spectrosc., 53, 150

Modigliani, A., Hummel, W., Abuter, R., et al. 2007, The SINFONI pipeline [arXiv: astro-ph/0701297]

Modigliani, A., Goldoni, P., Royer, F., et al. 2010, in Observatory Operations: Strategies, Processes, and Systems III, eds. D. R. Silva, A. B. Peck, \& B. T. Soifer, et al., Proc. SPIE, 7737, 773728

Moré, J. J., Garbow, B. S., \& Hillstrom, K. E. 1980, User Guide for MINPACK1, Argonne National Laboratory Report ANL-80-74, Argonne, Ill

Noll, S., Kausch, W., Barden, M., et al. 2012, A\&A, 543, A92

Parsons, S. G., Gänsicke, B. T., Marsh, T. R., et al. 2012, MNRAS, 426, 1950 Patat, F. 2008, A\&A, 481, 575

Rosenberg, M. J. F., van der Werf, P. P., \& Israel, F. P. 2012, A\&A, 540, A116

Rothman, L. S., Gordon, I. E., Barbe, A., et al. 2009, J. Quant. Spectrosc. Radiat. Transf., 110, 533

Rousselot, P., Lidman, C., Cuby, J.-G., Moreels, G., \& Monnet, G. 2000, A\&A, 354,1134

Taylor, M.J., Pendleton, W.R. Jr., Clark. S., et al. 1997, J. Geophys. Res., 102, 26283

van Rhijn, P. J. 1921, Publ. Astr. Lab. Groningen, 31, 1

Vernet, J., Dekker, H., D’Odorico, S., et al. 2011, A\&A, 536, A105 